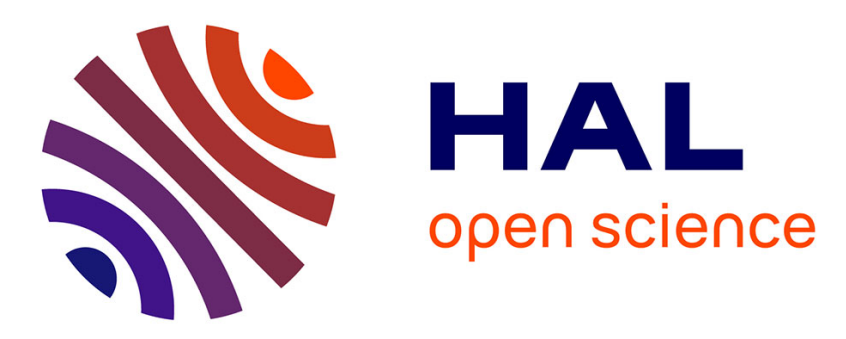

\title{
Calculation of the radiative properties of photosynthetic microorganisms
}

Jérémi Dauchet, Stéphane Blanco, Jean-François Cornet, Richard A Fournier

\section{To cite this version:}

Jérémi Dauchet, Stéphane Blanco, Jean-François Cornet, Richard A Fournier. Calculation of the radiative properties of photosynthetic microorganisms. Journal of Quantitative Spectroscopy and Radiative Transfer, 2015, 161, pp.60-84. 10.1016/j.jqsrt.2015.03.025 . hal-02698875

\section{HAL Id: hal-02698875 \\ https://hal.science/hal-02698875}

Submitted on 1 Jun 2020

HAL is a multi-disciplinary open access archive for the deposit and dissemination of scientific research documents, whether they are published or not. The documents may come from teaching and research institutions in France or abroad, or from public or private research centers.
L'archive ouverte pluridisciplinaire HAL, est destinée au dépôt et à la diffusion de documents scientifiques de niveau recherche, publiés ou non, émanant des établissements d'enseignement et de recherche français ou étrangers, des laboratoires publics ou privés. 


\title{
Calculation of the radiative properties of photosynthetic microorganisms
}

\author{
Jérémi Dauchet $^{\mathrm{a}, \mathrm{b}, *}$, Stéphane Blanco ${ }^{\mathrm{c}, \mathrm{d}}$, Jean-François Cornet $^{\mathrm{a}, \mathrm{b}}$, Richard Fournier ${ }^{\mathrm{c}, \mathrm{d}}$ \\ ${ }^{a}$ Clermont Université, ENSCCF, Institut Pascal - UMR 6602, BP 10448, F-63000 Clermont-Ferrand, France \\ ${ }^{b}$ CNRS, UMR 6602, IP, F-63171 Aubire \\ ${ }^{c}$ Université Paul Sabatier, UMR 5213 - Laboratoire Plasma et Conversion d'Energie (LAPLACE), Bât. 3R1, 118 route de \\ Narbonne, F-31062 Toulouse cedex 9, France \\ ${ }^{d}$ CNRS, LAPLACE, F-31062 Toulouse, France
}

\begin{abstract}
A generic methodological chain for the predictive calculation of the light-scattering and absorption properties of photosynthetic microorganisms within the visible spectrum is presented here. This methodology has been developed in order to provide the radiative properties needed for the analysis of radiative transfer within photobioreactor processes, with a view to enable their optimization for large-scale sustainable production of chemicals for energy and chemistry. It gathers an electromagnetic model of light-particle interaction along with detailed and validated protocols for the determination of input parameters: morphological and structural characteristics of the studied microorganisms as well as their photosynthetic-pigment content. The microorganisms are described as homogeneous equivalent-particles whose shape and size distribution is characterized by image analysis. The imaginary part of their refractive index is obtained thanks to a new and quite extended database of the in vivo absorption spectra of photosynthetic pigments (that is made available to the reader). The real part of the refractive index is then calculated by using the singly subtractive Kramers-Krönig approximation, for which the anchor point is determined with the Bruggeman mixing rule, based on the volume fraction of the microorganism internal-structures and their refractive indices (extracted from a database). Afterwards, the radiative properties are estimated using the Schiff approximation for spheroidal or cylindrical particles, as a first step toward the description of the complexity and diversity of the shapes encountered within the microbial world. Finally, these predictive results are confronted to experimental normal-hemispherical transmittance spectra for validation. This entire procedure is implemented for Rhodospirillum rubrum, Arthrospira platensis and Chlamydomonas reinhardtii, each representative of the main three kinds of photosynthetic microorganisms, i.e respectively photosynthetic bacteria, cyanobacteria and eukaryotic microalgae. The obtained results are in very good agreement with the experimental measurements when the shape of the microorganisms is well described (in comparison to the standard volume-equivalent sphere approximation). As a main perspective, the consideration of the helical shape of Arthrospira platensis appears to be a key to an accurate estimation of its radiative properties. On the whole, the presented methodological chain also appears of great interest for other scientific communities such as atmospheric science, oceanography, astrophysics and engineering.
\end{abstract}

Keywords: optical properties, radiative properties, light scattering, anomalous diffraction approximation, Schiff approximation, photosynthetic microorganisms, photobioreactor

\footnotetext{
*Corresponding author

Email address: jeremi.dauchet@univ-bpclermont.fr (Jérémi Dauchet)
} 


\section{Nomenclature}

[0] dimensionless

$c$ speed of light in the surrounding medium $[\mathrm{m} / \mathrm{s}]$

$c_{0}$ speed of light in vacuum $[\mathrm{m} / \mathrm{s}]$

$C_{p i g, j}$ concentration of the pigment species $j$ within the cell material $\left[\mathrm{kg} / \mathrm{m}^{3}\right]$

$C_{x}$ dry mass concentration within a culture $\left[\mathrm{kg} / \mathrm{m}^{3}\right]$

$E a_{p i g, j}$ absorption cross-section of the pigment species $j\left[\mathrm{~m}^{2} / \mathrm{kg}\right]$

$\mathbf{e}_{i}$ direction of incidence of radiation (unit vector)

$\mathbf{e}_{o}$ orientation of a particle (unit vector)

$\mathbf{e}_{s}$ scattering direction (unit vector)

$f_{j}$ volume fraction of the $j^{\text {th }}$ internal structure of a microorganism [0]

$k$ wave number of radiation $\left[\mathrm{m}^{-1}\right]$

$l$ crossing length of a ray passing through a particle $[\mathrm{m}]$

$M$ dry mass of a microorganism $[\mathrm{kg}]$

$m$ complex refractive index [0]

$n$ real part of the refractive index $[0]$

$n_{j}$ real refractive index of the $j^{\text {th }}$ internal structure of a microorganism [0]

$\mathcal{P}$ two-dimensional projected surface of a particle

$p_{X}$ probability density function of the random variable $X$

$R$ aspect ratio of a particle shape [0]

$r_{e q}$ radius of the volume equivalent sphere $[\mathrm{m}]$

$S$ complex scattering amplitude

$s$ width parameter of the log-normal distribution [0]

$\mathcal{D}$ tri-dimensional domain of the particle

$V_{p}$ volume of the particle $\left[\mathrm{m}^{3}\right]$

$W$ differential scattering cross-section $\left[\mathrm{m}^{2} / \mathrm{sr}\right]$ or $\left[\mathrm{m}^{2} / \mathrm{sr} / \mathrm{kg}\right]$

$w_{p i g, j}$ dry-mass fraction of the pigment species $j$ within the cell material [0]

$x$ size parameter $[0]$

$x_{w}$ intracellular water volume-fraction $[0]$

\section{Greek letters}

$\theta_{l}$ limit angle between small and large scattering angles (Schiff's approximation) [rad] 
$\theta$ polar angle $[\mathrm{rad}]$

$\kappa$ imaginary part of the refractive index $[0]$

$\lambda$ wavelength of radiation $[\mathrm{m}]$

$\lambda_{0}$ wavelength of radiation within vacuum $[\mathrm{m}]$

$\nu$ frequency of radiation $[\mathrm{Hz}]$

$\nu_{p}$ anchoring frequency of radiation (singly subtractive Kramers-Krönig approximation) $[\mathrm{Hz}]$

$\sigma$ cross-section $\left[\mathrm{m}^{2}\right]$ or $\left[\mathrm{m}^{2} / \mathrm{kg}\right]$

$\varphi$ azimuth angle $[\mathrm{rad}]$

\section{Subscripts/Superscripts}

$a$ refers to absorption

$e$ value within the surrounding medium

eff effective value (homogeneous equivalent or monodisperse equivalent)

ext refers to extinction

$r$ refractive indices relative to the surrounding medium

$s$ refers to scattering

$\nu$ value at the frequency $\nu$ of radiation

value for an isolated particle (fixed size and orientation)

- median or average value

Acronyms

DDA Discrete-Dipole Approximation

FDTD Finite Difference Time Domain

OD Optical Density

PAR Photosynthetically Active Radiation 


\section{Introduction}

Photosynthesis engineering is nowadays recognized as a possible alternative to the exhaustion of fossil resources and global warming. Indeed, it enables both the mitigation of $\mathrm{CO}_{2}$ emission and the sustainable synthesis of chemicals for energy (biomass, biofuels) as well as for chemistry (raw or high-value green products). Nevertheless, first the development of new and efficient technologies like photobioreactors devoted to the photosynthetic-microorganism cultivation with thermodynamic efficiencies approaching at least $10 \%$ is required. Such original future designs, or more basically the optimisation of existing concepts, are only feasible if predictive knowledge-models of these processes are developed on a strong physical basis, giving them sufficient genericity for industrial purposes (simulation, sizing, scale-up, optimization, model-based predictive control, etc). Assuming a proper mixing and that all physiological needs are maintained at their optimal conditions ( $\mathrm{pH}$, temperature, dissolved $\mathrm{CO}_{2}$, minerals), it has been clearly demonstrated in the past decades that photobioreactors are mainly governed by radiant light transfer inside the culture volume, determining the kinetic rates, energetic yields, biomass composition and pigment contents $[1,2$, $3,4,5,6,7,8]$. Accurate knowledge of the radiation fields is therefore required prior to any analysis of photosynthesis engineering, which is only feasible by properly solving the radiative transfer equation for any boundary condition, i.e any photobioreactor design $[1,9,8,10,11,12]$. This equation contains three parameters, respectively the absorption and scattering coefficients together with the scattering phasefunction, which must be known with accuracy if the resulting radiation-fields are envisaged to formulate a kinetic and energetic model of the reactor. Therefore, any radiative analysis of photobioreactors starts with the determination of the absorption and scattering properties of the involved photosynthetic-microorganisms. Yet this question is not trivial, and to the best of our knowledge, no available database provides the adequate spectral and angular information needed, even for the strains of microalgae that are currently widely cultivated. Obtaining these properties involves either highly specialized experiments $[13,14,15,16$, 17], or the construction of a model implying the resolution of the Maxwell's equations for particles with types of heterogeneities, sizes and shapes for which the usual numerical methods such as Lorenz-Mie, T-Matrix, FDTD, DDA, etc $[18,19]$ are yet impracticable in most cases. Within the international literature dedicated to the study of photobioreactors, L. Pilon's research group (University of California, Los Angeles) is the only one, to the best of our knowledge, that focuses on the experimental determination of the radiative properties of photosynthetic microorganisms, through single scattering experiments including the measurement of the angular distribution of the scattered power $[15,16,8]$. The present article addresses the electromagnetic modelling approach, and we hereafter present a methodological chain inherited from the expertise of the oceanography community, and more broadly, of all the communities that are confronted with the waveparticle interaction problem, in either atmospheric sciences, astrophysics or engineering. In this wide context, our concern is to account not only for the specificity of photosynthetic microorganisms, but also for the analysis and optimisation requirements of photobioreactor-engineering.

The above mentioned theoretical and experimental issues have gradually developed during the past 15 years, but yet they have remained overwhelmingly open-ended. In the light of this gap, numerous research groups have no alternatives but to make use of the empirical tools developed by the community over the past twenty years $[20,21,22,23,24,25,10]$. These tools are based on radically different modelling approaches from the one that we develop hereafter. The spacial dependence of radiative observable is indeed fixed a priori according to laws inspired by radiation physics (Beer-Lambert extinction law, hyperbolic laws, etc) that involve a set of free parameters to be adjusted, on a case by case basis, so as to conform to available experimental data. Of course these empirical approaches reach their limits when considering diverse operating modes of photobioreactors, due to the necessity to adjust the parameters for each of the studied situations (e.g reactor geometry, incident light flux and radiation frequency), or simply when considering a process with complex geometry (experimental data then being difficult to obtain). These are the constraints that have led us to identify the need for a predictive methodology, based on limited and "easily" accessible experimental parameters, allowing us to grasp the microorganisms' variability, from one species to another and as a function of the culture conditions (in particular as a function of the illuminating conditions) $[26,11,27]$. Within this framework, the purpose of the present article is to gather and extend our know-how in order to design a full methodological chain ranging from the determination of the input 
parameters of our model to the validation of its results.

In the following, we make use of several methods that are furthermore described, independently of each other, within the literature dedicated to light scattering by small particles $[28,29,30,31]$. The very general problem of modelling radiative properties is fully posed in these works, the formalism of which is here adopted with the conventions of [28] for the definition of complex amplitudes. We start, at Section 2, with an introduction to the specificity of our study through a detailed description of the shapes and internal structures of photosynthetic microorganisms. It then appears that the determination of radiative properties implies the construction of a model of the microorganism itself (its geometry and its internal features in terms of refractive index) as well as an implementation of the standard model of electromagnetism (resolution of Maxwell's equations). These two tasks are actually interlocked since even if a perfect description of the electromagnetic properties of the microorganisms was available, the resolution of Maxwell's equations would be impossible considering the current state of knowledge: to this date, the most typical internal heterogeneities and shapes correspond to ineluctable numerical difficulties. Therefore, we propose an approximate electromagnetic model that includes a simplification of the particles' description, the corresponding approximations being chosen in line with the requirements of our study. The first choice is the following: we refuse to make too much of a compromise on the information about shape, as we believe this characteristic to be essential regarding interaction with radiation, and to be at the source of what essentially distinguishes different species of microorganisms. On the other hand, we are willing to represent the internal heterogeneities thanks to the approximation of an effective homogeneous medium. Indeed, this approximation has been tested in many situations and appears to corrupt only the power scattered at large angles $[32,33,34,35]$. We retain it because a very small proportion of the incident power on a microorganism is backscattered ( $90 \%$ of the scattered power is usually contained within a solid angle of roughly $20^{\circ}$ aperture around the incident direction) and, for the targeted radiative configurations, backscattering has a limited influence: the few backscattered photons have a very limited impact on radiative transfer within the photobioreactor (which is not the case when analysing oceanic albedo for instance). Therefore, in order to be in a position to solve electromagnetism, we modify our description of the scatterers which become pseudo-homogeneous particles.

If the microorganisms we are interested in were all spherical, this simplification would be enough to enable the resolution of electromagnetism. But photosynthetic microorganisms have a great diversity of shapes, and the sphere is to the date the only finite-size shape for which Maxwell's equation can be solved within the whole range of size parameters (from 5 to 200 for microalgae). The effective medium approximation is therefore insufficient, and since we are keen on preserving the shape description, we are thus compelled to simplify the electromagnetic model of light-particle interaction. It is a common practice in the radiative transfer community to make use of simplifications corresponding to asymptotic approximations: Rayleigh approximation when size parameters approach zero, geometrical optics when size parameters approach infinity, etc. In the case of photosynthetic microorganisms, size parameters are large and refractive-index contrasts are low; we are at the tenuous particle limit corresponding to the validity range of the anomalous diffraction approximation, that we here revisit to extend its applicability, thanks to the Schiff approximation (see Section 3 and Appendix A). The conjunction of the effective medium approximation and the Schiff approximation makes our model numerically tractable, that is to say solvable with usual numerical methods that we detail in Section 4.2.

In the above description of our model and its numerical implementation (which is summarized in Table 1.a) there is no mention of a procedure leading to the determination of the shape, the effective spectral refractive indices and the size distribution of the microorganisms (regarding the distribution of orientations, isotropy is always retained due to the agitation that is needed for the mixing within the process). However, the purpose of the present article is the construction of a methodological chain consistent with the study of photobioreactors. The determination of these parameters is therefore a central issue: we must be in a position to grasp the operating mode dependence of these parameters, either from experimentally accessible data (within the scope of the photobioreactor engineers' practice), or from available databases. Our methodological chain thus implies following a set of characterization procedures (summarized in the Table 1.b) before any implementation of the previously formulated model. First, the shape and the size distribution of the microorganisms are determined thanks to optical microscopy and image analysis (see Section 4.1). 
Simple rotatory-symmetric parametric shapes are here retained, and the size distributions are modelled as log-normal distributions for the radius of the volume-equivalent sphere. The remaining procedures are dedicated to the determination of the particles' effective refractive index. Indeed, although the effective medium approximation is a significant simplification of the wave-particle interaction, what remains is the determination of the spectral properties of this equivalent homogeneous medium that must stand for the microorganisms' internal heterogeneities. First, we determine the imaginary part of the refractive index, which characterizes the absorption properties of the continuous medium constituting the microorganisms. Visible light absorption by microorganisms is considered to be exclusively due to the presence of photosynthetic pigments that are gathered within the photosynthetic antennae. The pigment molecules are diluted enough to be characterized by an in vivo absorption cross-section (independently of the microorganism species and the type of study), and an internal pigment concentration (that is, on the other hand, extremely dependent on the culture conditions). Additionally, within the spectral range of the PAR (Photosynthetically Active Radiation), for the microorganisms' internal characteristics (low variation of the real part of the refractive index and weak absorption within the particle), it is reasonable to consider the absorption properties of the equivalent homogeneous medium to be the ones that would be obtained if the pigment molecules were homogeneously diluted within the particles' volume. We therefore model the imaginary part of the effective refractive index by summing the absorption cross-sections of the pigment molecules (either available in the literature or measured by our research group, see Section 4.1 where we make a database available to the reader), each of these absorption spectra being weighted by the concentration of the corresponding pigment species within the addressed microorganism. To sum up:

- We designed, based on field proven protocols in microbiology, a procedure enabling the determination of the pigment composition (that is to say the concentration of each pigment species) of each microorganism, for each culture condition (see Section 4.1). These experimental protocols lead to the measurement of pigment composition in terms of the pigment's dry-mass fraction (i.e the microorganisms' mass fraction once their water has been extracted).

- Then, the volume fraction of intracellular water is used in order to obtain information about the internal pigment-concentrations.

The imaginary part of the refractive index is now determined, and the following procedures deal with its real part. The determination of the real part of the refractive index of the equivalent homogeneous medium is a major difficulty since, on the one hand very little information is available in the literature dedicated to photosynthetic microorganisms, and on the other hand, unlike the imaginary part, this effective property strongly depends on heterogeneities (within the studied spectral range). We therefore propose to apply the causality Kramers-Krönig relations (see Section 3), that formalize the redundancy of the information contained in the complex refractive index, and lead to an expression of the real part of the effective refractive index as a function of the spectrum of the imaginary part. If we had access to this imaginary part over the whole spectrum, Kramers-Krönig relations would be sufficient, but we only have access to a limited information since the previous procedure (protocols and physical reasoning) only concerned the determination of the imaginary part within the PAR spectral range. This is a usual situation and various practical solutions exist in order to compensate (at least partially) for this lack of information. We here use the singly subtractive Kramers-Krönig approximation that allows us to correct the results obtained from the information within the PAR alone thanks to the knowledge of the real part of the refractive index at a particular wavelength, that is the so-called anchor point (see Section 3). Therefore, what remains is the determination of the refractive index at this anchor point that is here chosen at a frequency for which the studied microorganism is non-absorbing (i.e dielectric, within the framework of the electromagnetic assumptions that we share with $[28,29,30,31])$. For this purpose we employ the Bruggeman mixing rule, which gives the effective refractive index of a composite particle based on the knowledge of the volume fraction and the refractive index of its different structures (see Section 3). The volume fractions of the structures are here obtained from electron microscopy and image analysis, or far less frequently, from the literature when therein available (see Section 4.1). Finally, a small database presented in Section 4.1 contains a certain amount of data that we were able to gather about the real index of microorganisms' internal structures, for 
frequencies at which they are dielectric. To sum up, the procedure for the determination of the real part spectrum of the refractive index of the microorganisms includes:

- the determination of the volume fraction of the internal structures from image analysis or literature data if available,

- the extraction of the real index of the structures from a database,

- the construction of the effective real index at the anchor point by using the Bruggeman mixing rule,

- and finally, the implementation of the singly subtractive Kramers-Krönig approximation.

In the former discussions we distinguished, for intelligibility, the light-particle interaction model from the procedures employed for the determination of the sizes, the shapes and the refractive index of the microorganisms. Nevertheless, it clearly appears that the procedures presented in the previous paragraph imply a set of modelling acts that have the exact same status as our light-particle interaction model. Therefore, we adopt the following outline for the present article:

- Section 3 gathers the entire model, including the formalization of the shapes, the sizes, the model of absorption by pigments, the use of the Bruggeman mixing rule, as well as the singly subtractive Kramers-Krönig approximation.

- Section 4.1 gathers all the know-how associated with the input parameters' determination, that is to say the setting of the parameters involved in the equation that describes the shape, the setting of the parameters involved in the size distribution, the measurement of the pigments' dry-mass fraction, of the intracellular-water volume fraction, of the internal structures' volume fractions, as well as the elaboration and utilization of the databases for the in vivo absorption spectra of pigments and for the refractive index of internal structures.

- Finally, Section 4.2 gathers the numerical methods used for the implementation of the methodological chain, up to the calculation of the radiative properties of a microorganism suspension.

In Section 6 our proposal extends further than the determination of these properties (Section 5), with the description of a validation procedure that is a true part of the methodological chain itself. Indeed, considering the interlocking between the steps described above, it remains necessary to use a control technique for the databases that we aim to produce and distribute. Yet again this procedure must be consistent with the object of study, implying in particular its accessibility for the bio-engineering community (through "wellknown" experimental protocols) and the production of a validation related to the study of radiative transfer within photobioreactors. This validation includes two steps:

- The measurement of the hemispherical transmittance of a microorganism suspension thanks to an optical setup equipped with an integrating sphere.

- Rigorous simulation using the Monte Carlo method (see Section 6.2) of the multiple-scattering hemispherical transmittance that would be measured if the microorganism suspension indeed possessed the scattering and absorption properties predicted in Section 5.

Comparing the experimental spectra with the simulated ones also allows us to perform a critical analysis of the sensitivity of our model to its input parameters (Section 6.3). In this way, we are also able to validate the value retained for the refractive index at the anchor point frequency (Section 6.4). 
a. Model

Light-particle interaction: scattering and absorption under the assump- General concepts tion of independent particles. $[28,29,30,31]$

Particle description: size, shape and internal structure. Section 2

Simplification of the heterogeneous internal structure: effective medium Section 3 approximation.

Modification of the light-particle interaction model: anomalous diffrac- Section 3 and Aption approximation revisited with the Schiff approximation. pendix A

Numerical implementation: integration of Schiff approximation, includ- Section 4.2 and [36] ing size-distribution and orientation-distribution.

b. Characterization chain

(1) Determination of the microorganism shape and size distribution thanks to image analysis (Section 4.1).

(2) Photosynthetic pigments extraction and pigment composition measurement (dry-mass fraction, see Section 4.1).

(3) Determination of the intracellular water volume-fraction (literature, specific protocols and use of the results of (1), see Section 4.1).

(4) Construction of the concentration of each pigment species contained within the microorganism from the results of (2) and (3) (Section 4.1).

(5) Construction of the imaginary part spectrum of the effective refractive index from the results of (4) and a database of the in vivo absorption spectra of the pigments (see Section 3 and Section 4.1 for the database).

(6) Determination of the volume fractions of the anatomic internal structures from image analysis (Section 4.1).

(7) Construction of the real part of the effective refractive index at the anchor point by using the Bruggeman mixing rule for the results of (6) and the internal-structure realindices extracted from a database (see Section 3 and Section 4.1 for the database).

(8) Construction of the real part spectrum of the effective refractive index by using the singly subtractive Kramers-Krönig approximation from the results of (5) and (7) (Section 3).

(9) Validation of the results obtained for the radiative properties thanks to an analysis of the hemispherical transmittance measured through a microorganisms suspension (see Section 6).

Table 1: Organization of the main elements of the methodological chain presented in the present article. 


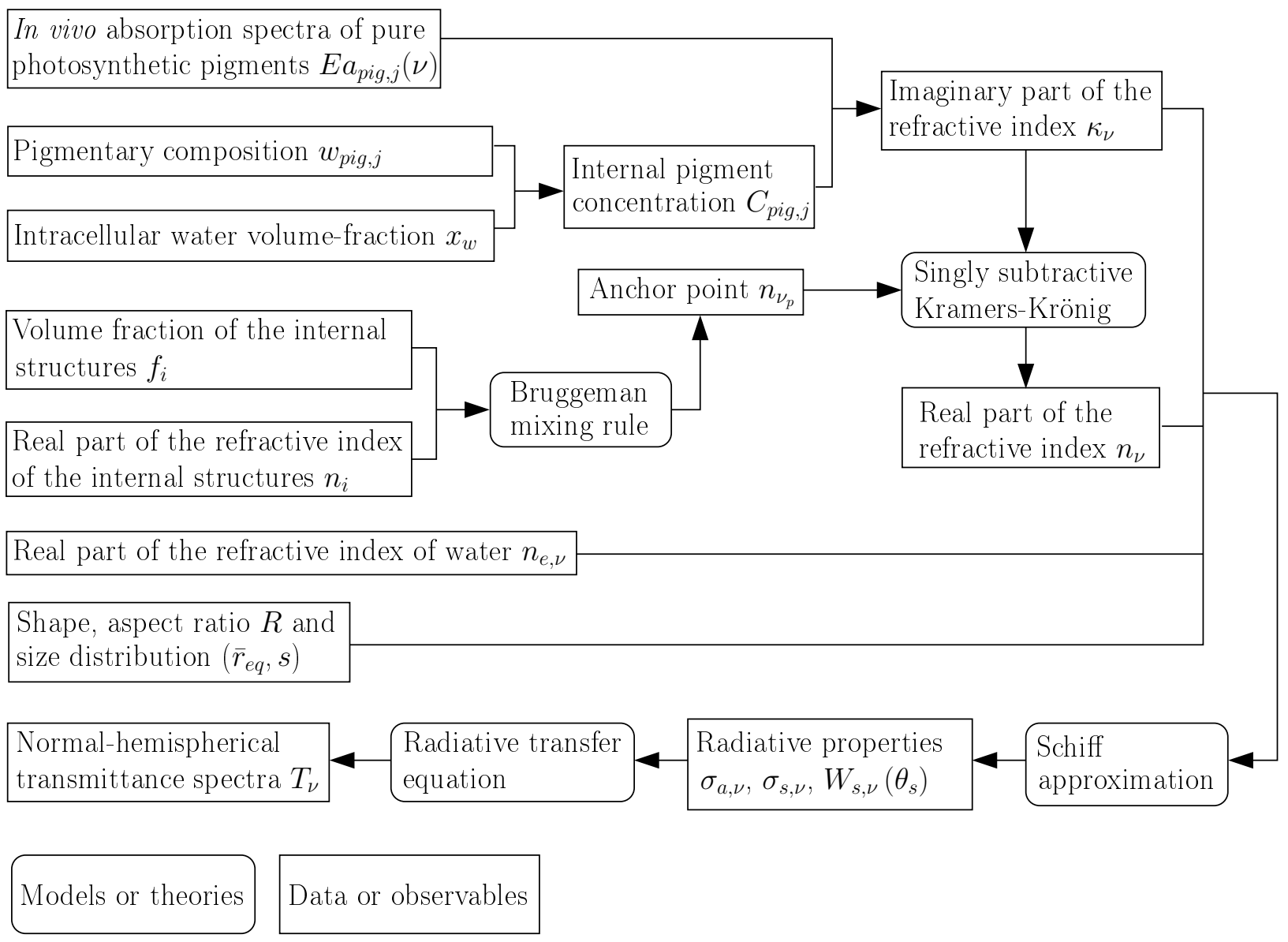

Figure 1: Summary of the presented methodological chain. 


\section{Photosynthetic microorganisms}

In this section, a quick overview of the morphological and structural characteristics of photosynthetic microorganisms is given with respect to the concerns of radiation physicists.

A fair number of microorganisms species have shapes that are close to rotatory symmetrical shapes such as cylinder, sphere, spheroid, super-spheroid, Tchebytchev particle, etc. The shapes of some species can significantly variate depending on the medium's chemical-composition and the culture conditions [37]. Typical characteristic sizes of photosynthetic microorganisms cultivated in photobioreactors range roughly from $1 \mu \mathrm{m}$ to $20 \mu \mathrm{m}$. Cultures of microorganisms of the same species have unimodal size and shape distributions corresponding to the diverse maturity-states of the cells. These size distributions are stationary, except in the very specific case of synchronized cultures. Microorganisms' shape and size is usually characterized by optical microscopy.

Beside their shape and size, the internal structure of microorganisms is an important feature that influences interaction with light. It corresponds to their anatomy and chemical composition, that determine their refractive index. Light absorption by photosynthetic microorganisms was the first radiative aspect to be investigated by oceanographers. Since then, it is considered that absorption by pigments is predominant [38, 39, 40, 41, 42]. Many different photosynthetic pigments exist, among which the most common groups are: Chlorophylls, Phycocyanins and Carotenoids. The measure of their spectral absorptionproperties under in vivo conditions corresponded to a research effort during the 70's and until the 90's [41]; fieldproven data and experimental protocols are now available in the literature. Each microorganism species has its own pigment composition corresponding to the respective proportion of the pigment types contained within the cell, that provides it with a unique radiative fingerprint. Some regulation mechanisms lead to the decrease of the total amount of pigment within the microorganism when it is highly irradiated, but the pigment composition, in the other hand, generally stays stable [7] (for standard photobioreactor culture-conditions). Yet again, numerous fieldproven protocols are available for the extraction and measure of the pigment composition. Typical pigment contents lead generally to absorption optical-depths inside microorganisms of the order of $10^{-2}$.

The microorganisms' anatomic structures are observable with optical microscopes working with visible wavelengths, implying that those heterogeneities play a role in the present study. At our level of description, the internal structures are extremely similar to those of other biological cells (except for the presence of photosynthetic pigments that is specific). The effective refractive index of biological cells was first investigated thanks to experiments such as immersion refractometry [43, 32] or spectroscopic inversion [44, 45, 46], providing with values ranging from 1.02 to 1.2 relative to water. More recently, in the trend of non-invasive medical-diagnosis and cytology, the tomography community developed microscopes dedicated to the measurement of the three-dimensional refractive index map of living cells [47, 48, 49,50,51, 52, 53, 54]. Those cartographies reveal relatively sharp variations of about $10 \%$ of the refractive index inside the cells. These variations draw the shape of the principal anatomic structures such as nucleus, mitochondria, etc, each of these structures representing from 1 to $10 \%$ of the cell volume. Microorganisms can therefore be pictured as composite material whose volume is divided into anatomic structures with relatively homogeneous refractive index. These internal structures are all nearly non-absorbing, except for the regions containing photosynthetic pigments, where the imaginary part of the refractive index is non-zero. The anatomic diversity of photosynthetic microorganisms is commonly represented by three groups that should be distinguished (see Fig. 2): bacteria, cyanobacteria, and eukaryotes (microalgae). Each of the three microorganisms that are studied in the present work represent one of these categories, with increasing intracellular complexity from simple bacteria with only cytoplasm, cell wall and reserves to the numerous structures of microalgae. We note that the refractive index of mitochondria has been shown to significantly variate depending on the metabolic state of living cells [49].

These results from the tomography community constitute a precious information but, to the best of our knowledge, only very few microorganisms and wavelengths have been investigated to date. Moreover, almost no quantitative information can be found about the number, the shape, the location and the size of the microorganisms internal structures. Therefore, the complexity associated with heterogeneity is one of the major issue for the radiative description of microorganisms. 

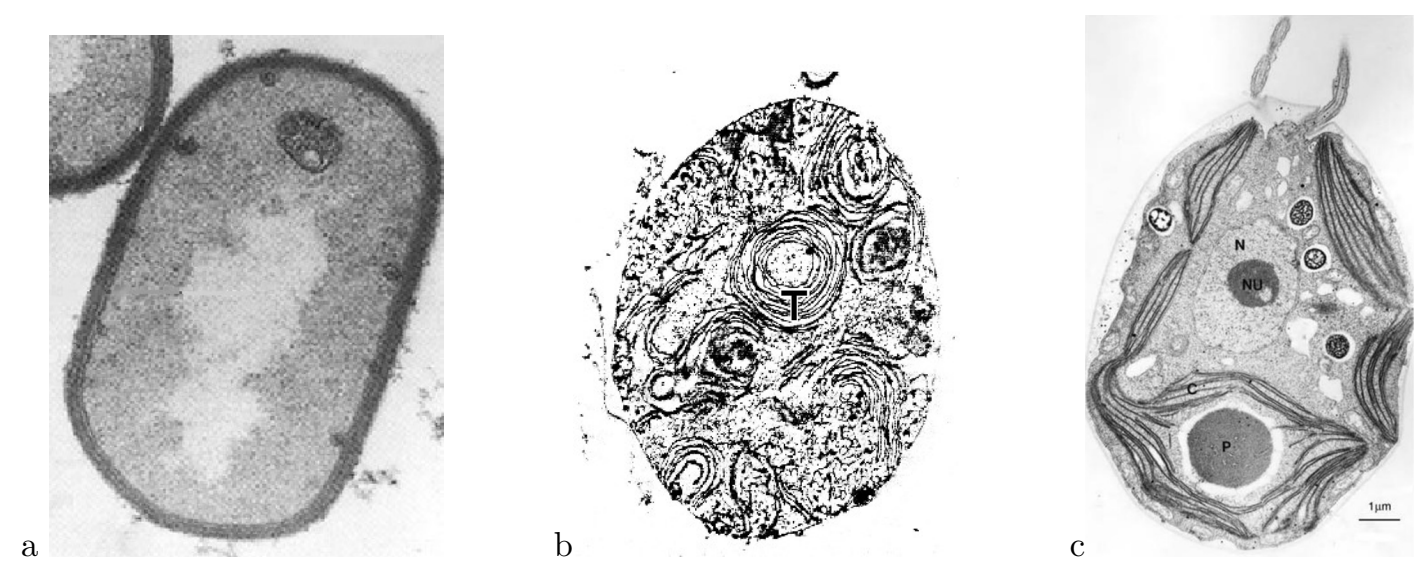

Figure 2: Anatomic diversity of photosynthetic microorganisms; increasing intracellular complexity from a to c. a: Photosynthetic bacteria (micrograph from [55]). b: Cyanobacteria (Transmission electron microscopy of a protoplast section of Arthrospira platensis, personal data); (T) Thylakoids, among others cellular features. c: Microalgae, i.e eukaryotic cell (median section of Chlamydomonas reinhardtii from [56]); (N) Nucleus, (Nu) Nucleolus, (C) Chloroplast, (P) Pyrenoid, among others cellular features.

\section{Model}

As stated earlier, the first step in the construction of our model consists in the assumption that microorganisms are homogeneous particles. The effective medium approximation defines an equivalent homogeneous medium leading to radiative properties that are "as close as possible" to the properties of the heterogeneous scatterer. This approximation is often applied to biological cells [32, 57, 39, 40], interstellar dusts [35, 58, 59] and atmospheric aerosols $[60,61,62]$. In the case of biological cells $[32,33,34]$, as in many other situations [35], it mainly produces errors on the energy scattered at large angles (i.e a very small proportion of the total energy scattered by microorganisms), which explains the successful use of this approximation for the study of microorganisms' cross-sections in oceanography. In the following, we therefore consider that microorganisms are homogeneous and we define a single complex refractive index $m_{\nu}^{\text {eff }}=n_{\nu}^{\text {eff }}-i \kappa_{\nu}^{\text {eff }}$ (where $\nu$ represents the spectral dependency of the index). Of course, the whole difficulty associated with the consideration of heterogeneities is then transferred to the definition of the $n_{\nu}^{e f f}$ and $\kappa_{\nu}^{e f f}$ spectra: we must ensure that the resolution of Maxwell's equations, for this effective particle, leads indeed to a satisfactory description of the interaction between light and the heterogeneous particle [30, 29, 63].

For the studied frequencies (i.e visible light) the imaginary part $\kappa_{\nu}$ of the refractive index is related to the absorption properties of photosynthetic pigments located within the photosynthetic antennas. Because these pigment-molecules are diluted, we make the assumption that each pigment interacts independently with radiation (this is a usual assumption in oceanography [38, 39, 40, 41, 42]). We can therefore define a molecular cross-section that characterizes the absorption properties of each pigment species independently of its concentration. Furthermore, if we accept that the pigment molecules are randomly distributed within the medium according to a uniform distribution (inside a small neighbourhood around the considered location; i.e continuous medium assumption), then the local value of the imaginary part of the refractive index is a function of molecular cross-sections and local concentrations:

$$
\kappa_{\nu}(\mathbf{r})=\frac{c_{0}}{4 \pi \nu} \sum_{j} \hat{C}_{p i g, j}(\mathbf{r}) E a_{p i g, j}(\nu)
$$

where $\hat{C}_{p i g, j}(\mathbf{r})$ is the concentration of the pigment species $j$ at location $\mathbf{r}$ in the cell-material volume and $E a_{p i g, j}(\nu)$ is the absorption cross-section per unit mass of the same pigment species under in vivo conditions. The spectrum of the in vivo absorption cross-section $E a_{p i g, j}(\nu)$ of many photosynthetic pigments are already published or have been established in this work (as detailed in Section 4.1) and they are the start basis for 
any predictive model of the radiative properties of photosynthetic microorganisms. Based on this local value $\kappa_{\nu}(\mathbf{r})$, the imaginary part of the equivalent homogeneous refractive index is usually calculated as the volume average of $\kappa_{\nu}(\mathbf{r})$ over the whole particle,

$$
\kappa_{\nu}^{e f f}=\frac{1}{V_{p}} \int_{\mathcal{D}} \kappa_{\nu}(\mathbf{r}) d \mathbf{r}=\frac{c_{0}}{4 \pi \nu} \sum_{j} C_{p i g, j} E a_{p i g, j}(\nu)
$$

where $V_{p}$ is the volume of the particle domain $\mathcal{D}$ and $C_{p i g, j}$ is the mass concentration of the pigment species $j$ within the microorganism cell-material, defined as:

$$
C_{p i g, j}=\frac{1}{V_{p}} \int_{\mathcal{D}} \hat{C}_{p i g, j}(\mathbf{r}) d \mathbf{r}
$$

The determination of the real part $n_{\nu}^{e f f}$ of the effective refractive index is a difficult task since this observable must stand for all the information associated with the microorganisms heterogeneities, in particular the spectra of $n_{\nu}$ for each of the internal anatomic structures. We hereafter partially bypass this difficulty by expressing $n_{\nu}^{e f f}$ as a function of the imaginary part spectrum $\kappa_{\nu}^{\text {eff }}$ (that we just modelled). This can be done by using the Ketteler-Helmholtz theory [29], that has been applied, among others, to photosynthetic microorganisms [40, 64], or the Kramers-Krönig relations [65, 66, 67], that have also been used for the study of photosynthetic microorganisms $[68,64]$ and other biological cells $[57,68]$ (as well as many more particle types). The following Kramers-Krönig relation is here applied to the effective properties of our microorganisms (see [67] Chap. 4.3.2 concerning the applicability of Kramers-Krönig to effective medium properties, and [64] for comparisons between the Ketteler-Helmholtz and Kramers-Krönig theories):

$$
n_{\nu}^{e f f}=1+\frac{2}{\pi} P \int_{0}^{+\infty} \frac{\nu^{\prime} \kappa_{\nu^{\prime}}^{e f f}}{\nu^{\prime 2}-\nu^{2}} d \nu^{\prime}
$$

where $P$ signifies that the Cauchy principal value has to be considered for the singularity $\left(\nu^{\prime}=\nu\right)$. The above relation (that is exact) consists in a spectral integration overs $[0,+\infty]$ while $\kappa_{\nu}^{\text {eff }}$ is only known in the visible and near infra-red region $\left[\nu_{\min }, \nu_{\max }\right]$. This situation is commonly encountered when applying the Kramers-Krönig relations to practical cases, and several approximations are available in order to compensate (at least partially) for this lack of spectral information. The most standard methods consist

- either in an extension of $\kappa_{\nu}^{e f f}$ outside the known spectrum, thanks to a model or measurements on similar materials [57],

- or in a transformation of Eq. 4 in order to bring out free parameters that are afterwards adjusted thanks to a set of frequencies at which $n_{\nu}^{e f f}$ is experimentally accessible [67].

Some approaches combine these two techniques [69].

In the present work, we make use of the singly subtractive Kramers-Krönig approximation [67]. The use of more advanced methods is an interesting perspective but it will require that models of $\kappa_{\nu}^{\text {eff }}$ outside the PAR develops and that experimental values of $n_{\nu}^{e f f}$ become more accessible. The singly subtractive Kramers-Krönig approximation is obtained by subtracting to Eq. 4 (for each $\nu$ ) the same equation taken at the particular frequency $\nu_{p}$ for which $n_{\nu_{p}}^{\text {eff }}$ is know. We thus ensure that the spectrum $n_{\nu}^{\text {eff }}$ takes the value $n_{\nu_{p}}^{e f f}$ at the frequency $\nu_{p}$, even when the integration is truncated to $\left[\nu_{\min }, \nu_{\max }\right]$ :

$$
n_{\nu}^{e f f}=n_{\nu_{p}}^{e f f}+\frac{2\left(\nu^{2}-\nu_{p}^{2}\right)}{\pi} P \int_{\nu_{\min }}^{\nu_{\max }} \frac{\nu^{\prime} \kappa_{\nu^{\prime}}^{\text {eff }}}{\left(\nu^{\prime 2}-\nu^{2}\right)\left(\nu^{\prime 2}-\nu_{p}^{2}\right)} d \nu^{\prime}
$$

This way, most of the complexity associated with the treatment of the microorganisms heterogeneity is reduced to the anchor point, i.e to the knowledge of the effective monochromatic refractive index $n_{\nu_{p}}^{\text {eff }}$ at the frequency $\nu_{p}$ only. 
We now have to construct a model for $n_{\nu_{p}}^{e f f}$, in agreement with our current state of knowledge of the heterogeneities. For this purpose, we retain a frequency $\nu_{p}$ at which the photosynthetic microorganism is dielectric $\left(\kappa_{\nu_{p}}^{e f f} \simeq 0\right)$ in order to simplify the access to the properties of the internal anatomic structures. Indeed, as mentioned in Section 2, the only distinction between photosynthetic microorganisms and other living cells is the absorption of radiation (of course not from biological point of view but only as far as our study is concerned). At frequency $\nu_{p}$, we therefore consider that the properties of the internal structures of photosynthetic microorganisms are identical to those of other living cells [70], which allows us to seek the needed information within a much wider literature. But going through this literature, we unfortunately faced the fact that biologists have only little interest in the properties that we seek: basically volume fractions and refractive index spectra of nucleus, cell wall, cytoplasm, etc. In order to make the best use of the available information, our approach is the following:

- When we find in the literature results of refractive index measurements for anatomic structures of photosynthetic organisms, the investigated frequencies are, for practical reasons, frequencies at which the microorganism is non-absorbing, that is to say close to our anchor point. We therefore directly use them.

- When we find in the literature results of refractive index measurements for similar anatomic structures of non-photosynthetic organisms, then, since they are dielectric over the whole optical spectrum, the authors had no reasons to investigate a frequency that is close to our anchor point. Nevertheless, the spectral variations of the refractive index of non-photosynthetic organisms are far less important than those of their photosynthetic equivalents $[57,43]$ and we do not hesitate to consider that these measurements give the same results as if they were performed at our anchoring frequency.

At Section 4.1 we construct, from a literature review, a small database containing the real part of the refractive index of various anatomic structures. Using this database, we now have to explore the several relations that express the effective refractive index of a composite material (here $n_{\nu_{p}}^{e f f}$ ) as a function of its internal structure (see [30, 29,63] for an overview). These so-called mixing rules are applied, among others, to the study of scatterers such as interstellar dusts [35, 58, 59], atmospheric aerosols [60, 61, 62, 71, 72], phytoplankton [43], etc. They usually correspond to a given topology of the composite material, and a set of approximations which implications are often not well understood [30, 60, 35, 58, 59, 61]. Standard mixing rules such as Lorentz-Lorenz, Maxwell-Garnett, and Bruggeman's relations are derived under the assumption that heterogeneities are very small compared to the wavelength of incident radiation, which is not the case for photosynthetic microorganisms. There is an ongoing search for the construction of extended mixing rules allowing larger sizes of inclusions [30], but in the present context, considering the lack of reliability of the information about internal structures and the unavoidable errors associated with the use of a mixing rule in the case of such complex particles as microorganisms, we propose to use the Bruggeman mixing rule Eq. 6 that is simple to implement, well documented, and compatible with our limited topological-knowledge since its derivation assumes uniformly distributed heterogeneities without specific shapes:

$$
\sum_{j} f_{j} \frac{\left(n_{j, \nu_{p}}\right)^{2}-\left(n_{\nu_{p}}^{e f f}\right)^{2}}{\left(n_{j, \nu_{p}}\right)^{2}+2\left(n_{\nu_{p}}^{e f f}\right)^{2}}=0
$$

where $f_{j}$ and $n_{j, \nu_{p}}$ are respectively the volume fraction and the real part of the refractive index of the $j^{\text {th }}$ internal structure of the studied microorganism. Moreover, we compared the values of $n_{\nu_{p}}^{e f f}$ given by the Bruggeman and the Maxwell-Garnett mixing rules for many microorganisms and we always recorded extremely similar results. We invite the reader wishing to explore the principles of Bruggeman's mixing-rule to refer to $[30,63]$. The use of more advanced mixing rules is an interesting improvement of our model that will be possible if topological-knowledge of microorganisms develops.

At this stage, using the singly subtractive Kramers-Krönig approximation Eq. 5 for which the anchor point is determined with Eq. 6, we have a model for the complex refractive index of the effective particle and we can define the relative refractive index $m_{r, \nu}$

$$
m_{r, \nu}=n_{r, \nu}-i \kappa_{r, \nu}=\frac{n_{\nu}^{e f f}}{n_{e, \nu}}-i \frac{\kappa_{\nu}^{e f f}}{n_{e, \nu}}
$$


where the aqueous surrounding medium is here considered to be dielectric with same refractive index $n_{e, \nu}$ as water. This relative index will be used for the calculation of the radiative properties, but first, the size and the shape of the particle have to be modelled.

The size distribution of the studied microorganisms, within a culture, is modelled (in agreement with experimental observations) as a $\log$-normal distribution $p_{R_{e q}}\left(r_{e q}\right)$ of the volume-equivalent sphere radius $r_{e q}$ :

$$
p_{R_{e q}}\left(r_{e q}\right)=\frac{1}{\sqrt{2 \pi} r_{e q} \ln (s)} \exp \left[-\frac{\left(\ln r_{e q}-\ln \bar{r}_{e q}\right)^{2}}{2 \ln (s)}\right]
$$

where $\bar{r}_{e q}$ is the median value and $s$ represents the "width" of the distribution. We define the effective radius $r_{e q}^{e f f}$ (see [28]) that will be occasionally used in order to represent practically this distribution as an equivalent monodisperse-distribution (in particular for the calculation of the microorganisms dry mass):

$$
r_{e q}^{e f f}=\frac{\int_{0}^{\infty} p_{R_{e q}}\left(r_{e q}\right) \pi r_{e q}^{2} r_{e q} d r_{e q}}{\int_{0}^{\infty} p_{R_{e q}}\left(r_{e q}\right) \pi r_{e q}^{2} d r_{e q}}=\bar{r}_{e q} \exp \left(\frac{5}{2} l n^{2}(s)\right)
$$

It must be clearly noticed at this stage that any other distribution function, or actual experimental distribution, can be alternatively used in the following.

The geometry of the outside envelop is a crucial information in our context, since it considerably determines our ability or not to solve Maxwell's equations (now that the issue of the heterogeneities has been bypassed thanks to a description in terms of an effective homogeneous medium). Most of the available radiative-properties models make use of the equivalent sphere approximation (see [46, 39, 40,64,32] for microorganisms but this practice extends way beyond this context). In this case, the question of light-particle interaction modelling is not an issue since the sphere is a shape for which Maxwell's equations are easily and rigorously solved for almost any size (reliable open sources codes are available and computation times are extremely reasonable). Let us briefly discuss the principle of the volume-equivalent sphere approximation that will be hereafter retained as a baseline for our results involving other shapes. Under this approximation, microorganisms are homogeneous spheres of radius $r_{e q}$ distributed according to Eq. 8 and Maxwell's equations can be solved using any Lorenz-Mie code (see Section 4.2). The equivalent sphere is usually defined as having same volume (or surface-area) as the nonspherical scatterer, which generates significant errors in the two following cases $[73,74,75]$ :

1. the fact that the sphere has same volume or surface as the nonspherical scatterer is not a pertinent criterion of equivalence

2. there is no sphere giving radiative properties with sufficient accuracy for photobioreactor-engineering, even with more advanced criterion of equivalence than volume or surface.

We therefore propose a model that includes a further accurate description of the microorganisms shape in order to tackle the various and complex morphologies encountered in the microbial world. The issue of how accurate this geometric description must be is still an open question and we aim here to give part of the answer by considering, as a first investigation, simple rotatory symmetric shapes such as cylinders (see Eq. 10) and spheroids (see Eq. 11).

$$
\begin{aligned}
& \rho(\varphi, z)=a, \text { for }-b<z<b \\
& R=\frac{a}{b} \\
& r_{e q}=a\left(\frac{3}{2 R}\right)^{\frac{1}{3}}
\end{aligned}
$$

where the above cylinder parametric equation is expressed in the cylindrical coordinate system $(\rho, \varphi, z)$ with $\rho \in[0,+\infty], \varphi \in[0,2 \pi]$ and $z \in[-\infty,+\infty]$.

$$
\begin{aligned}
& r(\theta, \varphi)=\left\{\left|\frac{1}{a} \sin (\theta)\right|^{2}+\left|\frac{1}{b} \cos (\theta)\right|^{2}\right\}^{-\frac{1}{2}} \\
& R=\frac{a}{b} \\
& r_{e q}=a\left(\frac{1}{R}\right)^{\frac{1}{3}}
\end{aligned}
$$


where the above spheroid parametric equation is expressed in the spherical coordinate system $(r, \theta, \varphi)$ with $r \in[0,+\infty], \theta \in[0, \pi]$ and $\varphi \in[0,2 \pi]$. Therefore, a new parameter is now necessary (besides $r_{e q}$ ) for the definition of the scatterer shape: the aspect ratio ${ }^{1} R$. The distribution of $R$ over the microorganism culture is neglected hereafter (i.e $R$ is the average aspect ratio), which is a common approximation in the literature devoted to non-spherical scatterers [76, 29, 77, 28, 30,31, 78], including microorganisms [45, 44] (see [74] for a discussion on the consequence of this approximation).

It must be noticed that if the present work focuses on the study of cylindrical and spheroidal shapes, it is not a restriction of the method, but it has been decided by the authors for the following reasons:

- This paper already deals with numerous concepts and original results, and considering more complex geometries would require the description of advanced numerics that are out of the scope of the present text (they will appear in a separate publication).

- Moreover, the theoretical derivations of the absorption and scattering cross sections that we propose hereafter have been already obtained independently in the literature, in the framework of the anomalous diffraction approximation for spheroids and cylinders. Therefore, no doubt can exist on the formula used in this work, except for phase function derivation (see hereafter).

- Finally, two microorganisms (over the three that are investigated in this study) have shapes that are very close to cylinders (or rods) and spheroids, so this choice constitutes already a strong improvement when compared to the sphere.

Now that the scatterers are completely defined (by their refractive index and their geometry), we focus on the analysis of their interaction with light. Various techniques are available in order to solve the problem of an electromagnetic wave scattered by an homogeneous particle. Each of these techniques is limited by the range of geometries and refractive indices that can be tackled [18, 19, 28, 29, 30, 31]. Identifying those that are appropriated for the study of photosynthetic microorganisms (from pragmatic point of view) implies to investigate the available formal solutions (including approximations) and numerical methods enabling their implementation, considering that

1. the size parameter $x=\frac{2 \pi r_{e q}}{\lambda_{e}}$ (with $\lambda_{e}$ the wavelength of incident radiation in the surrounding medium) of microorganisms cultivated in photobioreactors ranges from 5 to 200 ,

2. many microorganisms are strongly elongated particles that can be up to 50 times longer than wide, implying very small radius of curvature,

3. microorganisms have complex refractive index corresponding to low dielectric contrast with respect to their surrounding medium $\left(n_{r, \nu} \in[1.02,1.2], \kappa_{r, \nu} \in\left[10^{-5}, 10^{-2}\right]\right)$.

The above description corresponds to an identified situation for which very little can be done to the date (see [74] in the context of mineral dusts, [76] for ice crystals, and [30] for an overview). The computation of the rigorous solution of Maxwell's equations is indeed usually obtained thanks to numerical methods that, to our knowledge, fail to tackle the conjunction of the first two above listed criteria. The methods consisting in a division of the particle volume or surface into discrete elements or sources (see $[18,19]$ and the cited references, see also [30] for the most popular of those methods) are limited by the too high number of elements required for most microorganisms (regarding available memory size and access speed). As for the methods based on a functional decomposition of the electromagnetic field, such as T-Matrix [30, 31], Lorenz-Mie [28, 29], and other similar methods (e.g [79]), they are limited by the current floating point accuracy. The ongoing search for the development of these methods $(e . g[18,80])$ suggests that it will eventually become possible to rigorously solve Maxwell's equations for most microorganisms, but to date, it is still necessary to employ approximations. The above listed two first criteria are out of the validity range of both the Rayleigh approximation $(x \ll 1)$ and the geometrical-optics approximation $(x \gg 1$ and radius of curvature much larger than the wavelength), but hopefully the low dielectric contrast of microorganisms

\footnotetext{
${ }^{1}$ The aspect ratio is here chosen as the inverse of the elongation: $R<1$ for elongated particles, $R>1$ for flattened particles.
} 
(i.e criteria 3.) leads to important simplifications. It corresponds to the Rayleigh-Gans approximation for low size-parameters, and the anomalous diffraction approximation or the Schiff approximation for large size-parameters $[28,29,31,81]$.

The anomalous diffraction approximation was introduced in 1957 by Van de Hulst [28]. It is still widely used and subject to further developments [82, 73, 83, 84]. It is associated with the two following validity conditions (conditions that define the so-called soft particles):

- $x \gg 1$,

- $\left|m_{r}-1\right| \ll 1$.

This approximation, whose accuracy has been investigated in various context (see e.g [85, 86]), is identified as appropriated to the study of nonspherical soft particles such as ice crystals [76, 30], fractal aggregates [78], and especially biological cells [82, 40, 39, 87].

The anomalous diffraction approximation is based on physical pictures that are only valid for small scattering angles. It therefore gives the absorption and scattering cross-sections, as well as the differential scattering cross-section for the directions around the incident direction, but it does not enable a complete angular description. This issue is well identified (see [40] for example) but little addressed in the literature ${ }^{2}$. For typical sizes and refractive indices encountered in this work, around $90 \%$ of the total power scattered by a microorganism belongs to the angular range tackled by the anomalous diffraction approximation, and although the approximation is not valid for large angles, we recorded surprisingly accurate results when applied over $[0, \pi]$, even including a fair estimate of the asymmetry parameter. Therefore, the use of the anomalous diffraction approximation could be sufficient (except in the specific case of single-backscattering, that is essential for remote-sensing studies but that is out of the scope of our study). Nevertheless, the calculation times for the evaluation of the differential scattering cross-section at large angle are very long: the calculation of the single scattering phase function for one wavelength takes up to 10 days ${ }^{3}$ (for the most critical situations, i.e highest size-parameters and lowest aspect ratios, that are for example encountered in the study of Arthrospira platensis, see Fig. 5). These calculation times are not compatible with the necessity for our study to construct a spectral database including several microorganisms with several physiological states (determining the input parameters of our model) that vary with culture conditions (e.g variation of the pigment-content in the case of mineral starvation for biofuel production). This calculation-time issue lead us to investigate the Schiff approximation [81], i.e the quantum mechanics equivalent of the anomalous diffraction approximation, including a specific description of scattering at large angles. We try to argue here that Schiff's approximation is to date the best available model for the study of photosynthetic microorganisms, since it allows to account for their elongated and complex shapes, within their range of size parameters, including an angular description of the backscattered power. Unfortunately, the evaluation of the Schiff approximation at large angle leads to the exact same numerical difficulties as for the anomalous diffraction approximation. Schiff's approximation has the further advantage of being physically justified for backward directions but it is still numerically impractical. Nevertheless, the corresponding formalism has new useful mathematical properties. We hereafter propose to rework the Schiff's expression accordingly, in order to extract a simplified but fully practical description of large-angle scattering. We retain this simplified model as a novel proposition, but we keep in mind that it remains approximate and that the determination of the backscattering properties of tenuous particles remains an open question. Finally, we note that when studying particularly small microorganisms, other methods can be substituted to the Schiff approximation (T-Matrix, Rayleigh-Gans, DDA, etc) without any modification of the whole methodological chain that is proposed in the present article.

\footnotetext{
${ }^{2}$ Relatively recent works propose formulations of the single scattering phase function (and therefore of the differential scattering cross-section at any angle) from the anomalous diffraction approximation [83, 84], but unfortunately no available demonstration is associated with these results.

${ }^{3}$ Specific codes developed with Matlab 6.5 and based on numerical quadratures. The integrals of the anomalous diffraction approximation are solved by using the quad8 function (high order adaptive Simpson's rule), the integration over the size distribution is solved by using the quadl function, and the integration over the orientations is solved by using a Gaussian quadrature of order 24. These results have been obtained with a Xeon $2.8 \mathrm{GHz}$ biprocessor, without parallelization.
} 
The Schiff approximation is further detailed in [81] and our large-angle developments are given at Appendix A; we hereafter only present the fundamental physical pictures and give the resulting general expressions. Schiff's approximation is associated with the same two validity criteria as for the anomalous diffraction approximation, and with the definition of an angle $\theta_{l}$ that sets the limit between small and large scattering angles [81]:

$$
\theta_{l}=\sqrt{\frac{2}{\tilde{x}}}
$$

where $\tilde{x}$ is the size parameter as calculated for the smallest characteristic dimension of the scatterer (this dimension controls the angular aperture of the scattered power). For the small scattering angles $\left(\theta_{s}<\theta_{l}\right)$, the approximation corresponds to simple physical pictures that are associated with its validity conditions:

- $x \gg 1$, that ensures that the ray picture of the geometrical optics is applicable,

- $\left|m_{r}-1\right| \ll 1$, that ensures that the reflection and refraction of incident light by the particle can be ignored: the incident rays cross the particle in straight line, they do not bend when entering and leaving the particle.

Then, the approximation is based on the premise that extinction is the result of the interference between the rays that are attenuated and phase shifted by crossing the particle and the rays that do not pass through the particle. On the other hand, absorption is due to the attenuation of light along the straight lines passing through the particle. Therefore, the mathematical expressions of the radiative properties correspond to a simple integration of the attenuations and phase shifts due to the crossing length through the particle at each location of its projected surface seen from the incident direction. The interference phenomena lead to an expression similar to that of the Fraunhofer diffraction pattern ${ }^{4}$. For an isolated particle with a fixed orientation $\mathbf{e}_{\mathbf{o}}$ (with respect to the incidence $\mathbf{e}_{\mathbf{i}}$ of the plane wave) and a size represented by the radius $r_{e q}$ of the volume equivalent sphere, the expression for the scattering amplitude is the following:

$$
\begin{gathered}
\hat{S}_{\nu}\left(\mathbf{e}_{s}, \mathbf{e}_{i} ; \mathbf{e}_{o}, r_{e q}\right)=\frac{k_{e, \nu}}{2 \pi} \iint_{\mathcal{P}\left(\mathbf{e}_{o}, r_{e q}\right)} d x d y \exp \left[i k_{e, \nu} \theta_{s}\left(x \cos \varphi_{s}+y \sin \varphi_{s}\right)\right] \quad, \theta_{s}<\theta_{l} \\
\times\left\{1-\exp \left[-i k_{e, \nu} l\left(x, y ; \mathbf{e}_{o}, r_{e q}\right)\left(m_{r, \nu}-1\right)\right]\right\}
\end{gathered}
$$

The cross-sections are therefore:

$$
\begin{array}{ll}
\hat{\sigma}_{e x t, \nu}\left(\mathbf{e}_{o}, r_{e q}\right)=\frac{4 \pi}{k_{e, \nu}} \operatorname{Re}\left\{\hat{S}_{\nu}\left(\mathbf{e}_{i}, \mathbf{e}_{i} ; \mathbf{e}_{o}, r_{e q}\right)\right\} & \text { (Optical theorem) } \\
\hat{\sigma}_{a, \nu}\left(\mathbf{e}_{o}, r_{e q}\right)=\iint_{\mathcal{P}\left(\mathbf{e}_{o}, r_{e q}\right)} d x d y\left[1-\exp \left(-2 k_{e, \nu} l\left(x, y ; \mathbf{e}_{o}, r_{e q}\right) \kappa_{r, \nu}\right)\right] \\
\hat{\sigma}_{s, \nu}\left(\mathbf{e}_{o}, r_{e q}\right)=\hat{\sigma}_{e x t, \nu}\left(\mathbf{e}_{o}, r_{e q}\right)-\hat{\sigma}_{a, \nu}\left(\mathbf{e}_{o}, r_{e q}\right) \\
\hat{W}_{s, \nu}\left(\mathbf{e}_{s} \mid \mathbf{e}_{i} ; \mathbf{e}_{o}, r_{e q}\right)=\left|\hat{S}_{\nu}\left(\mathbf{e}_{s}, \mathbf{e}_{i} ; \mathbf{e}_{o}, r_{e q}\right)\right|^{2}, \quad \theta_{s}<\theta_{l}
\end{array}
$$

where $\hat{\sigma}_{e x t}, \hat{\sigma}_{a}, \hat{\sigma}_{s, \nu}$ are respectively the extinction, absorption and scattering cross-sections and $\hat{W}_{s}$ is the differential scattering cross-section, with Re the real part, and $k_{e, \nu}=\frac{2 \pi}{\lambda_{e, \nu}}$ the wave number within the surrounding medium of refractive index $n_{e, \nu}$ where the wavelength of the incident wave is $\lambda_{e, \nu}=\frac{\lambda_{0}}{n_{e, \nu}}, \lambda_{0}$ being the wavelength in vacuum. $\mathcal{P}\left(\mathbf{e}_{o}, r_{e q}\right)$ is the projected surface of the particle seen from the incident direction and $l\left(x, y ; \mathbf{e}_{o}, r_{e q}\right)$ is the crossing-length of an incident ray intersecting $\mathcal{P}$ at location $(x, y)$. Both

\footnotetext{
${ }^{4}$ Interferences in the far field zone are expressed as a first order Taylor expansion around $\theta_{s}=0$, which allows to reduce the integration over the particle volume to an integration over its geometric section $\mathcal{P}$. See [81] for more details.
} 
$\mathcal{P}$ and $l$ are a function of the particle orientation $\mathbf{e}_{o}$ and size $r_{e q}$. Notations are defined in Fig. 3. We note that in the above expression of $\hat{\sigma}_{e x t, \nu}\left(\mathbf{e}_{o}, r_{e q}\right)$, the angle $\theta_{s}$ is zero since $\hat{S}_{\nu}$ is taken for the forward direction $\mathbf{e}_{\mathbf{s}}=\mathbf{e}_{\mathbf{i}}$, which gives a very simple expression:

$$
\hat{\sigma}_{e x t, \nu}\left(\mathbf{e}_{o}, r_{e q}\right)=2 \operatorname{Re}\left\{\iint_{\mathcal{P}\left(\mathbf{e}_{o}, r_{e q}\right)} d x d y\left\{1-\exp \left[-i k_{e, \nu} l\left(x, y ; \mathbf{e}_{o}, r_{e q}\right)\left(m_{r, \nu}-1\right)\right]\right\}\right\}
$$

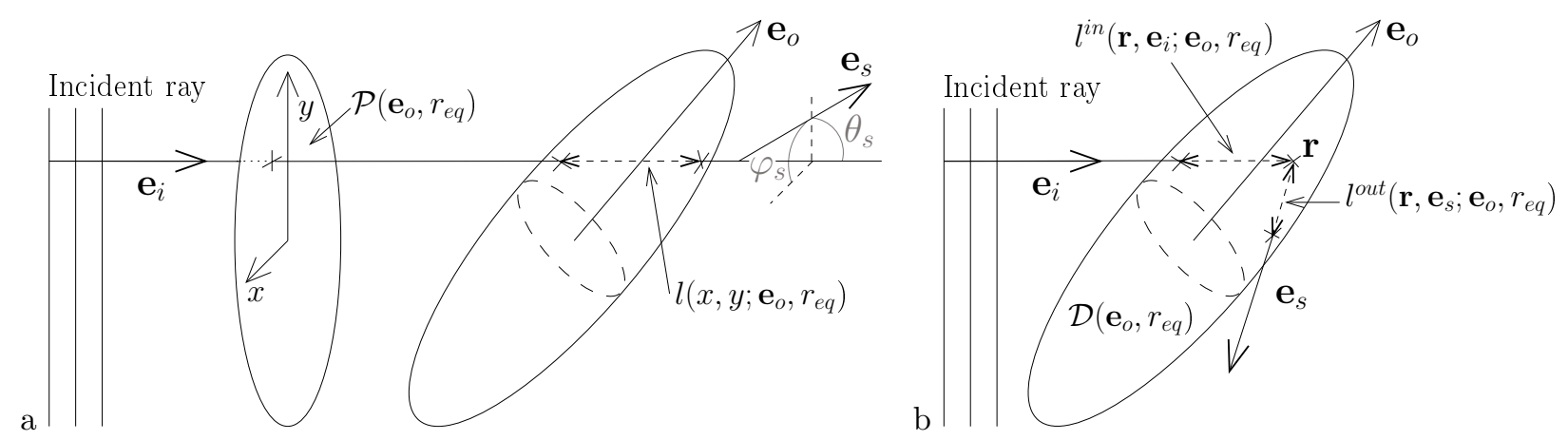

Figure 3: Definition of the notations used for Schiff's approximation: spheroid with size $r_{e q}$ (illustrating rotationally axisymetric particles) whose axis of revolution is along $\mathbf{e}_{o}$. The incident direction is $\mathbf{e}_{i} . \mathcal{P}\left(\mathbf{e}_{o}, r_{e q}\right)$ is the projected surface of the particle in a plane perpendicular to $\mathbf{e}_{i}$. a: $\mathcal{P}$ is associated with the referential $(x, y)$. The scattering direction $\mathbf{e}_{s}$ makes an angle $\theta_{s}$ with $\mathbf{e}_{i}$ and $\varphi_{s}$ with the axis $x . l\left(x, y ; \mathbf{e}_{o}, r_{e q}\right)$ is the crossing length of a ray collinear to $\mathbf{e}_{i}$ passing through the particle in straight line and intersecting $\mathcal{P}$ at location $(x, y)$. Analytic expression of $l\left(x, y ; \mathbf{e}_{o}, r_{e q}\right)$ for cylinders and spheroids is given in [82]. b: $l^{i n}\left(\mathbf{r}, \mathbf{e}_{i} ; \mathbf{e}_{o}, r_{e q}\right)$ is the length of an incident ray (with direction $\left.\mathbf{e}_{i}\right)$ between its entry into the particle and the location $\mathbf{r}$ within the particle volume $\mathcal{D}\left(\mathbf{e}_{o}, r_{e q}\right) ; l^{\text {out }}\left(\mathbf{r}, \mathbf{e}_{s} ; \mathbf{e}_{o}, r_{e q}\right)$ is the length of a ray starting from location $\mathbf{r}$, with the scattering direction $\mathbf{e}_{s}$, until it exits the particle.

For large angles, the physical pictures remains closely similar but the deviation of optical paths by the particle can no longer be neglected. This deviation is not due to the refraction and reflection phenomena at the interface between the particle and the surrounding medium (we stay within the same pictures as before) but it is due to the interaction between the incident radiation and the material constituting the particle. The scattering amplitude is expressed as the sum of all the attenuations and phase shifts along incident rays in direction $\mathbf{e}_{i}$, that interact at a location $\mathbf{r}$ within the particle and go out with the scattering direction $\mathbf{e}_{s}$. The integration domain can no longer be reduced to the projected surface of the particle and it is now performed over its volume $\mathcal{D}$ :

$$
\begin{aligned}
\hat{S}_{\nu}\left(\mathbf{e}_{s}, \mathbf{e}_{i} ; \mathbf{e}_{o}, r_{e q}\right) & =\frac{i k_{e, \nu}^{2}}{2 \pi}\left(m_{r, \nu}-1\right) \int_{\mathcal{D}\left(\mathbf{e}_{o}, r_{e q}\right)} d \mathbf{r} \exp (i \mathbf{q} \cdot \mathbf{r}) \\
& \times \exp \left\{-i k_{e, \nu}\left(m_{r, \nu}-1\right)\left[l^{i n}\left(\mathbf{r}, \mathbf{e}_{i} ; \mathbf{e}_{o}, r_{e q}\right)+l^{\text {out }}\left(\mathbf{r}, \mathbf{e}_{s} ; \mathbf{e}_{o}, r_{e q}\right)\right]\right\}
\end{aligned}
$$

where $\mathbf{q}=k_{e, \nu}\left(\mathbf{e}_{s}-\mathbf{e}_{i}\right)$ and the other notations are defined in Fig. 3. Because the numerical evaluation of this integral is very tedious and time consuming (as explained above), we tried to develop a good approximation for the direct calculation of $\left|\hat{S}_{\nu}\right|^{2}$. This approximation is presented here for the first time, contrary to the other parts of our model (that are all well-identified in the literature), and it will be further discussed in subsequent works focussing on backscattering properties of soft particles. Yet, this approximation belongs to the present state-of-the-art methodology for computing microorganisms' radiative properties. First, we show in Appendix A that we can retain $|\hat{S}|^{2} \propto \frac{1}{\sin ^{3}\left(\theta_{s} / 2\right)} \frac{1+\cos ^{2}\left(\theta_{s}\right)}{2}$ as a first approximation of Eq. 16 . On this basis, we propose to furthermore deteriorate the backscattering model as $|\hat{S}|^{2}=\frac{r}{\sin ^{n}\left(\theta_{s} / 2\right)} \frac{1+\cos ^{2}\left(\theta_{s}\right)}{2}$ in order to establish a model with two free parameters $r$ and $n$ that allow to fulfil the continuity of $W_{s}$ at $\theta_{l}$, the normalization $\sigma_{s}=\int_{4 \pi} d \mathbf{e}_{s} W_{s}\left(\mathbf{e}_{s} \mid \mathbf{e}_{i}\right)$ and $W_{s}>0$, without degrading the results of the Schiff approximation 
for small angles. Indeed, it is important here to not deteriorate the description of the small angles since they are essential for our study and since they correspond to a better level of approximation. We note that all the situations treated in Section 5 correspond to values of $n$ that are close to 3 (more precisely $2<n<4$ ), as in the expression of $|\hat{S}|^{2}$ before deterioration. After averaging over the orientation distribution $p_{\mathbf{E}_{\mathbf{o}}}\left(\mathbf{e}_{\mathbf{o}}\right)$ and the size distribution $p_{R_{e q}}\left(r_{e q}\right)$, our model of the scattering by a microorganisms suspension (under the assumption of independent scattering) is:

$$
\begin{gathered}
\sigma_{e x t, \nu}=\int_{4 \pi} d \mathbf{e}_{o} p_{\mathbf{E}_{o}}\left(\mathbf{e}_{o}\right) \int_{0}^{\infty} d r_{e q} p_{R_{e q}}\left(r_{e q}\right) \hat{\sigma}_{e x t, \nu}\left(\mathbf{e}_{o}, r_{e q}\right) \\
\sigma_{a, \nu}=\int_{4 \pi} d \mathbf{e}_{o} p_{\mathbf{E}_{o}}\left(\mathbf{e}_{o}\right) \int_{0}^{\infty} d r_{e q} p_{R_{e q}}\left(r_{e q}\right) \hat{\sigma}_{a, \nu}\left(\mathbf{e}_{o}, r_{e q}\right) \\
\sigma_{s, \nu}=\sigma_{e x t, \nu}-\sigma_{a, \nu} \\
W_{s, \nu}\left(\theta_{s}\right)= \begin{cases}\int_{4 \pi} d \mathbf{e}_{o} p_{\mathbf{E}_{o}}\left(\mathbf{e}_{o}\right) \int_{0}^{\infty} d r_{e q} p_{R_{e q}}\left(r_{e q}\right) \hat{W}_{s, \nu}\left(\mathbf{e}_{s} \mid \mathbf{e}_{i} ; \mathbf{e}_{o}, r_{e q}\right) & , \theta_{s} \leq \theta_{l} \\
\frac{r}{\sin ^{n}\left(\theta_{s} / 2\right)} \frac{1+\cos ^{2}\left(\theta_{s}\right)}{2} & , \theta_{s} \geq \theta_{l}\end{cases}
\end{gathered}
$$

where, considering the mixing required for photobioreactor to operate at optimal culture-conditions, the orientation distribution is isotropic $p_{\mathbf{E}_{\mathbf{o}}}\left(\mathbf{e}_{\mathbf{o}}\right)=\frac{1}{4 \pi}$ over the whole solid angle (that is here noted $\left.4 \pi\right), p_{R_{e q}}\left(r_{e q}\right)$ is defined at Eq. 8, $\hat{\sigma}_{e x t, \nu}\left(\mathbf{e}_{o}, r_{e q}\right), \hat{\sigma}_{a, \nu}\left(\mathbf{e}_{o}, r_{e q}\right), \hat{W}_{s, \nu}\left(\mathbf{e}_{d} \mid \mathbf{e}_{i} ; \mathbf{e}_{o}, r_{e q}\right)$ are given at Eqs. 13 and 14, and where the two parameters $r$ and $n$ of the model at large angles are adjusted to fulfil the two following conditions:

1. $W_{s, \nu}$ is continuous at the limit angle:

$$
\frac{r}{\sin ^{n}\left(\theta_{l}\right) / 2} \frac{1+\cos ^{2}\left(\theta_{l}\right)}{2}=\int_{4 \pi} d \mathbf{e}_{o} p_{\mathbf{E}_{o}}\left(\mathbf{e}_{o}\right) \int_{0}^{\infty} d r_{e q} p_{R_{e q}}\left(r_{e q}\right) \hat{W}_{s, \nu}\left(\theta_{l}, \varphi_{s} ; \mathbf{e}_{o}, r_{e q}\right)
$$

where $\hat{W}_{s, \nu}\left(\mathbf{e}_{s} \mid \mathbf{e}_{i} ; \mathbf{e}_{o}, r_{e q}\right)$ is written as a function of the two angles $\theta_{s}$ and $\varphi_{s}$ defined in Fig. 3 in order to specify that the integration is performed at $\theta_{s}=\theta_{l}$,

2. and the scattering cross-section $\sigma_{s, \nu}$ Eq. 17 is the integral of $W_{s, \nu}$ over the whole solid angle:

$$
2 \pi \int_{0}^{\pi} d \theta_{s} \sin \left(\theta_{s}\right) W_{s, \nu}\left(\theta_{s}\right)=\sigma_{s, \nu}
$$

where the integration of $W_{s, \nu}\left(\theta_{s}\right)$ is analytic for $\theta_{s} \geq \theta_{l}$.

The construction of our model is now complete. This model is based on a set of input parameters that are: the in vivo absorption spectra of the pigment molecules, the pigment composition of the microorganisms, the real part of the refractive index of the internal structures at any wavelength for which the microorganism is dielectric, the volume fraction of these internal structures, and finally the size distribution and the average aspect ratio of the microorganisms. In the next section we present the experimental protocols and the databases that we currently use for the determination of these input parameters.

\section{Practical implementation}

\subsection{Determination of the input parameters of the model}

Except for the database of the pigment's in vivo absorption spectra, parameters that are discussed in this section depend on the culture conditions (the most sensitive is the pigment composition). The culture conditions that are studied in Section 5 are standard subculture conditions: shaked $250 \mathrm{~mL}$ erlenmeyer, 100 $\mathrm{rpm}$, low photon flux density around $30 \mu \mathrm{mol}_{h \nu} \cdot \mathrm{m}^{-2} \cdot \mathrm{s}^{-1}\left(\simeq 7 \mathrm{~W} \cdot \mathrm{m}^{-2}\right)$, optimal $\mathrm{pH}$ and temperature. In the 
following, the protocols for the determination of these parameters are presented and discussed. The values retained for the studied standard-conditions are gathered at Section 5, in the caption of Figs. 4, 5 and 6. If different culture conditions are considered, the parameters have to be measured (or found in literature) accordingly.

The absorption spectra $E a_{\text {pig }}(\nu)$ of pure in vivo pigments are the key input data of the present methodology. Absorption properties of pigments are strongly dependent on their surrounding environment inside the microorganism (which we name "in vivo conditions", in line with the pioneer literature [41]):

- Pigments are associated with proteins within the so-called antenna pigment-protein complexes, where energy-transfer coupling between molecules is strong. As a consequence, the width of the absorption rays are significantly enlarged and microorganisms' absorption spectra are smooth continuum [88, 38].

- The previous molecular edifices are surrounded by intracellular water, that is the main constitutivecomponent of cytoplasm.

Two distinct approaches for the measure of the pigment's in vivo properties are found in the literature. The first method consists in an inversion of absorption spectra of microorganism suspensions, or remote sensing data $[40,38,39]$. In this case, the difficulty is to account for the scattering by microorganisms and to distinguish between the contribution of different pigments. In other hand, we are sure that the measure is performed under in vivo conditions. The second method consists in the quantitative measurement of the absorption spectra of pure pigments in water or organic solvents (depending of their solubility). In this case, the difficulty is to ensure actual in vivo conditions. Indeed, since many isolated pigments are not water soluble, the use of organic solvent is sometime unavoidable (typically for chlorophylls or carotenoids). These organic solvents can lead to spectral shifts up to $40 \mathrm{~nm}$ for carotenoids or $100 \mathrm{~nm}$ for bacteriochlorophylls. This effect is accounted for by correcting the obtained pigment-spectra on the basis of microorganism absorption-spectra [41].

In the present work, it is important that the predictive determination of pigments' absorption properties be independent of the model of scattering by microorganisms. Therefore, the second method has been extensively used, according to the pioneer work of [41]. We constructed a database gathering 14 among the most important photosynthetic pigments in nature (representatives of photosynthetic bacteria, cyanobacteria and microalgae), that is made available in Supplementary Material [89]. Data for chlorophyll $a$, photosynthetic carotenoids and photoprotective carotenoids, that all require organic solvents for extraction, are extended and slightly modified values of [41] (according to our experimental results). Data for chlorophylls $b$ and $c$, two other pigments requiring organic solvent, are directly obtained from interpolation of [41], with an extension over the range 350 to $400 \mathrm{~nm}$. Seven water-soluble pigments are also provided in the database: Y-phycoerythrin has been interpolated from [41]; R-phycoerythrin, B-phycoerythrin, phycoerythrocyanin, C-phycocyanin, R-phycocyanin and allophycocyanin are own experimental data of the authors. Finally, two other pigments requiring organic solvent, that are encountered within the purple photosynthetic bacterium Rhodospirillum rubrum (the bacteriochlorophyll $a$ and the total carotenoids), have also been measured by the authors. This database is central in the present study and it aims at contributing to the development of knowledge models of photosynthetic microorganisms in general.

The pigment composition $w_{\text {pig,j }}$ is determined thanks to two distinct measurements:

1. the pigment concentration within a sample of microorganism suspension,

2. the total dry-biomass concentration within the same sample (weighted after drying of a sample in oven, $110^{\circ} \mathrm{C}, 24 \mathrm{~h}$ ).

$w_{p i g, j}$ is the ratio of the result of measurement 1 over the result of measurement 2 . This measure of $w_{p i g, j}$ corresponds a major experimental incertitude of our methodology (accuracy around 15\%), which will be investigated in Sections 5 and 6 . The pigment concentration (measurement 1 ) is obtained using two different approaches depending on the solvent properties. For pigments that are soluble in organic solvent, a definite volume of microalgal suspension is centrifugated (5000 g, $5 \mathrm{~min}, 4^{\circ} \mathrm{C}$ ) and the supernatant evacuated. A known volume of appropriate solvent is then added and extraction is performed until the complete depigmentation of the cells. The sample is centrifugated again, in the same conditions, and the optical density 
(OD) of the supernatant is measured at specific wavelengths (spectrophotometer SAFAS, MC2, Monaco). For water-soluble pigments, a known volume of microalgal suspension is frozen and defrosted seven times (in liquid nitrogen at $77 \mathrm{~K}$ ), then centrifugated $\left(10000 \mathrm{~g}, 5 \mathrm{~min}, 4^{\circ} \mathrm{C}\right.$ ) before reading ODs of the supernatant. In every case, the pigment concentrations are obtained by using well-established correlations from the literature. These correlations give the pigment concentrations with acceptable accuracy, as a function of the ODs at some specific wavelengths. This standard and convenient method avoids operating a chromatographic separation of each pigment, which remains the reference procedure if any doubt arises about the OD correlations. For Arthrospira platensis, we used OD440, 620 and $678 \mathrm{~nm}$ with the correlations of Myers and Kratz [90] for water-soluble pigments and Bennet and Bogorad [91] for solvent-soluble pigments. For Chlamydomonas reinhardtii, all pigments are solvent-soluble and we used OD480, 630, 645, 665, $750 \mathrm{~nm}$ with the correlation of Strickland and Parsons [92]. Finally, for the solvent-soluble pigments of Rhodospirillum rubrum, we used OD515, 720 and $880 \mathrm{~nm}$ with the correlations of Vernon and Garcia [93].

Once $w_{p i g, j}$ is measured, the concentration $C_{p i g, j}$ of each pigment species $j$ within the whole microorganism volume (the cell material) is deduced from:

$$
C_{p i g, j}=w_{p i g, j} \rho_{d r y}\left(1-x_{w}\right)
$$

where $\rho_{d r y}$ is the density of the dry biomass, that is a remarkably constant property of microorganisms: $\rho_{d r y}=1400 \pm 100 \mathrm{~kg} \cdot \mathrm{m}^{-3}[94,95]$, and where $x_{w}$ is the intracellular-water volume fraction, that is also a relatively constant property for any microorganism cultivated in standard conditions, without $\mathrm{pH}$ or osmotic effects [96, 45]: $x_{w}=0.80 \pm 0.04$.

Real part of the refractive index of internal structures of microorganisms for the calculation of the anchor point: the small up-to-date database presented in Table 2 gathers the information found in the literature for different structures of bacteria, cyanobacteria and eukaryotic microalgae. The values that are used in the present work are also indicated. The correlation reported in [97] has been used for the spectral refractive index $n_{e, \nu}$ of water (i.e the surrounding medium of the microorganisms).

\begin{tabular}{|c|c|c|c|c|c|c|c|c|c|}
\hline \multirow{2}{*}{\multicolumn{2}{|c|}{$\begin{array}{l}\text { Group } \\
\text { Structure }\end{array}$}} & \multicolumn{3}{|c|}{ Bacteria $[44,45,46]$} & \multicolumn{5}{|c|}{ Cyanobacteria (vegetative cell ${ }^{d}$ ) $[53]$} \\
\hline & & Wall $^{c}$ & Cytoplasm & $\mathrm{PHB}^{e}$ & Wall $^{c}$ & Thyla & roid & Nucleoid & Cytoplasm $^{b}$ \\
\hline \multicolumn{2}{|l|}{$n$} & $1.40-1.42$ & $1.37-1.38$ & $1.45[98]$ & 1.39 & 1.4 & & 1.58 & \\
\hline \multicolumn{2}{|c|}{ Value used } & 1.41 & 1.375 & 1.45 & 1.39 & 1.4 & & 1.58 & 1.36 \\
\hline \multirow{2}{*}{$\begin{array}{l}\text { Groupe } \\
\text { Structure }\end{array}$} & \multicolumn{9}{|c|}{ Eukaryotes } \\
\hline & \multicolumn{2}{|r|}{ Wall $^{c}$} & Nucleolus & Nucleus $^{a}$ & \multicolumn{2}{|c|}{ Chloroplast } & \multicolumn{2}{|c|}{ Mitochondria } & Cytoplasm \\
\hline \multirow[t]{3}{*}{$n$} & \multirow{3}{*}{\multicolumn{2}{|c|}{$\begin{array}{c}1.36-1.53[43] \\
1.5[32]\end{array}$}} & $1.38[48]$ & $1.36[48]$ & \multirow{3}{*}{\multicolumn{2}{|c|}{$\begin{array}{c}1.36-1.40[43] \\
1.38[48] \\
1.51[32]\end{array}$}} & \multirow{3}{*}{\multicolumn{2}{|c|}{$1.41-1.43[48]$}} & $1.36-1.39[51]$ \\
\hline & & & $1.38[51]$ & $1.36[51]$ & & & & & $1.34[53]$ \\
\hline & & & $1.44[53]$ & $1.36[53]$ & & & & & $1.35[32]$ \\
\hline $\begin{array}{l}\text { Value } \\
\text { used }\end{array}$ & \multicolumn{2}{|r|}{1.5} & 1.40 & 1.36 & \multicolumn{2}{|c|}{1.51} & \multicolumn{2}{|r|}{1.42} & $1.36^{b}$ \\
\hline
\end{tabular}

Table 2: Real part of the refractive index of different microorganism internal-structures, for visible and near-infrared wavelengths. When in the literature refractive index for various metabolic states is reported, we retain here the state corresponding to typical growing-subculture conditions. ${ }^{a}$ Other parts than the nucleolus. ${ }^{b}$ Rest of the cytoplasm. ${ }^{c}$ cytoplasmic membrane, cell wall and external layers. $d$ heterocysts and akinete being ignored. $e$ Polyhydroxybutyrate stock. Microorganisms cultivated within well-mixed photobioreactors generally do not contain vacuoles. Yet, when target organisms contain vacuoles, the refractive index of oxygen $(n=1)$ should be retained for these structures.

The volume fraction $f_{j}$ of internal structures of microorganisms (for the division defined in Tab. 2) is determined from micrographs, on a case by case basis. Very little information are found in the literature about this structural division of the volume, and for most microorganisms there is no other choice than to use rough estimations. Hopefully, detailed information on Chlamydomonas reinhardtii are available from [99].

The size and the shape of microorganisms is determined by two-dimensional image processing. The most appropriate shape for a given microorganism is determined by the experimenter from microscope observation. Then, a set of micrographs obtained with an optical microscope (Olympus BX41, Japan) linked to a CCD 
camera (Kappa, PS 30) is analyzed with the Image Pro Plus software (Media Cybernetics, USA) in order to measure the distribution of the aspect ratio and the distribution of the volume-equivalent sphere radius $r_{e q}{ }^{5}$. The two parameters $\bar{r}_{e q}$ and $s$ of the modelled log-normal size-distribution Eq. 8 are adjusted in order that $p_{R_{e q}}\left(r_{e q}\right)$ fits the experimental distribution. Finally, the average aspect ratio $R$ (averaged over the set of micrographs) is retained.

Values measured for $w_{p i g, j}, f_{j}, \bar{r}_{e q}, s, \mathrm{R}$ and values retained for $x_{w}$, in the case of the studied microorganisms under standard subculture-conditions, are reported in the captions of Figs. 4, 5, and 6 .

Finally, an intermediate validation of $x_{w}, C_{x}$ and size-distribution is performed, based on the following relation that must be verified for a given sample of microorganism suspension:

$$
\frac{C_{x}}{N_{p}}=M_{e f f}=\bar{V}_{p} \rho_{d r y}\left(1-x_{w}\right)
$$

where $C_{x}$ is the dry-biomass weight of the sample, $\rho_{d r y}$ is the dry-biomass volumetric mass density (constant value, see above), $1-x_{w}$ is the intracellular dry-biomass volume fraction, $N_{p}$ is the number of microorganisms per unit volume of the sample (experimentally obtained by using a Malassez cell), and $\bar{V}_{p}$ is the microorganism average-volume calculated with the size distribution (sometime approximated to $4 \pi / 3\left(r_{e q}^{e f f}\right)^{3}$, with $r_{e q}^{e f f}$ defined at Eq. 9). $\frac{C_{x}}{N_{p}}$ is measured for many samples with different concentrations $\left(\bar{V}_{p}, x_{w}\right.$ and $\rho_{d r y}$ are independent of the concentration), and we verify that its average value $\overline{\left(\frac{C_{x}}{N_{p}}\right)}$ satisfies $\overline{\left(\frac{C_{x}}{N_{p}}\right)}=\bar{V}_{p} \rho_{d r y}\left(1-x_{w}\right) \pm 10 \%$, which ensures the self-consistency of the model's input parameters $\left(x_{w}, C_{x}, \bar{V}_{p}\right)$ and appears as a crucial validation step in the experimental methodology developed along with this article.

\subsection{Numerical methods}

The numerical implementation of the model includes the following steps:

- The anchor point satisfying the Bruggeman mixing rule is determined using a Newton method.

- The Single Subtractive Kramers-Krönig approximation is implemented thanks to a Matlab 6.5 code provided in [67].

- The equivalent sphere model is solved thanks to the Lorenz-Mie code provided in [31].

- The integrals of the Schiff approximation (Eqs. 13, 14, 17 and 18) are solved by Monte Carlo integration, as reported in [36]. Identical results were obtained with quadrature integration, but we recorded higher calculation times.

- The parameters $n$ and $r$ involved in the expression of the differential scattering cross-section at large angles (see Eq. 18) are identified thanks to a Newton method insuring the two conditions Eqs. 19 and 20 .

\footnotetext{
${ }^{5}$ The observations are performed under typical subculture conditions
} 


\section{Results for refractive indices and radiative properties}

Results obtained with our model (using the Schiff approximation for an accurate treatment of the scatterer's shape) and its comparison with the volume-equivalent sphere approximation (using standard rigorous Lorenz-Mie calculation) are given in Fig. 4 for the purple bacterium Rhodospirillum rubrum, in Fig. 5 for the cyanobacterium Arthrospira platensis, and Fig. 6 for the eukaryotic microalga Chlamydomonas reinhardtii. The scattering and absorption cross-sections are expressed in $\mathrm{m}^{2}$ per $\mathrm{kg}$ of dry biomass, thanks to a division of the particulate cross-sections by the effective dry-mass $M_{\text {eff }}$ of one microorganism particle ${ }^{6}$ (see Eq. 22). The errors associated with the incertitude on the measure of the pigment composition (which is identified as the main experimental error) are hereafter investigated by reporting the results obtained for the maximum and minimum values of $w_{p i g, j}$ as errorbars, assuming monotony ${ }^{7}$.

These results confirm two expected behaviours. First, we confirm that single-scattering phase functions are greatly oriented in the forward directions where the scattering diagram is sensitive to the particle shape. Second, the absorption cross-sections obtained with an equivalent-sphere are very close to those obtained with a modelled shape when the refractive index of the particle is close to that of water, or when the shape of the particle is close to that of a sphere : a significant deviation occurs only for Arthrospira platensis. At the opposite, the values of the scattering cross-section are always sensitive to the shape of the scatterer, leading to very important deviations between the models (except for Chlamydomonas reinhardtii that is quasi-spherical).

\footnotetext{
${ }^{6}$ The resolution of the light-scattering problem leads to cross-sections $\sigma$ expressed in $m^{2}$ per particle. Considering the mass $M$ (in $\mathrm{kg}$ ) of this particle, one can define the cross-section $\tilde{\sigma}=\sigma / M$ that is expressed in $\mathrm{m}^{2}$ per $\mathrm{kg}$ of particles. When dealing with radiative transfer (see Section 6.2), working with such mass cross-sections can be extremely convenient if the mass of particles within the studied suspension is easier to evaluate than their number. In the present context, the microorganism content of a suspension is commonly analysed in terms of its dry-mass, that is its mass once water have been evaporated: only the dry-biomass of the microorganisms is weighted. For this reason, it is a common practice in the photobioreactor community to devide the cross-sections $\sigma$ by the effective dry mass $M_{e f f}$ of the microorganisms.

${ }^{7}$ For the sake of legibility, errorbars are only reported onto the model accounting for the microorganism shape, but similar relative errors are obtained with the equivalent-sphere model.
} 

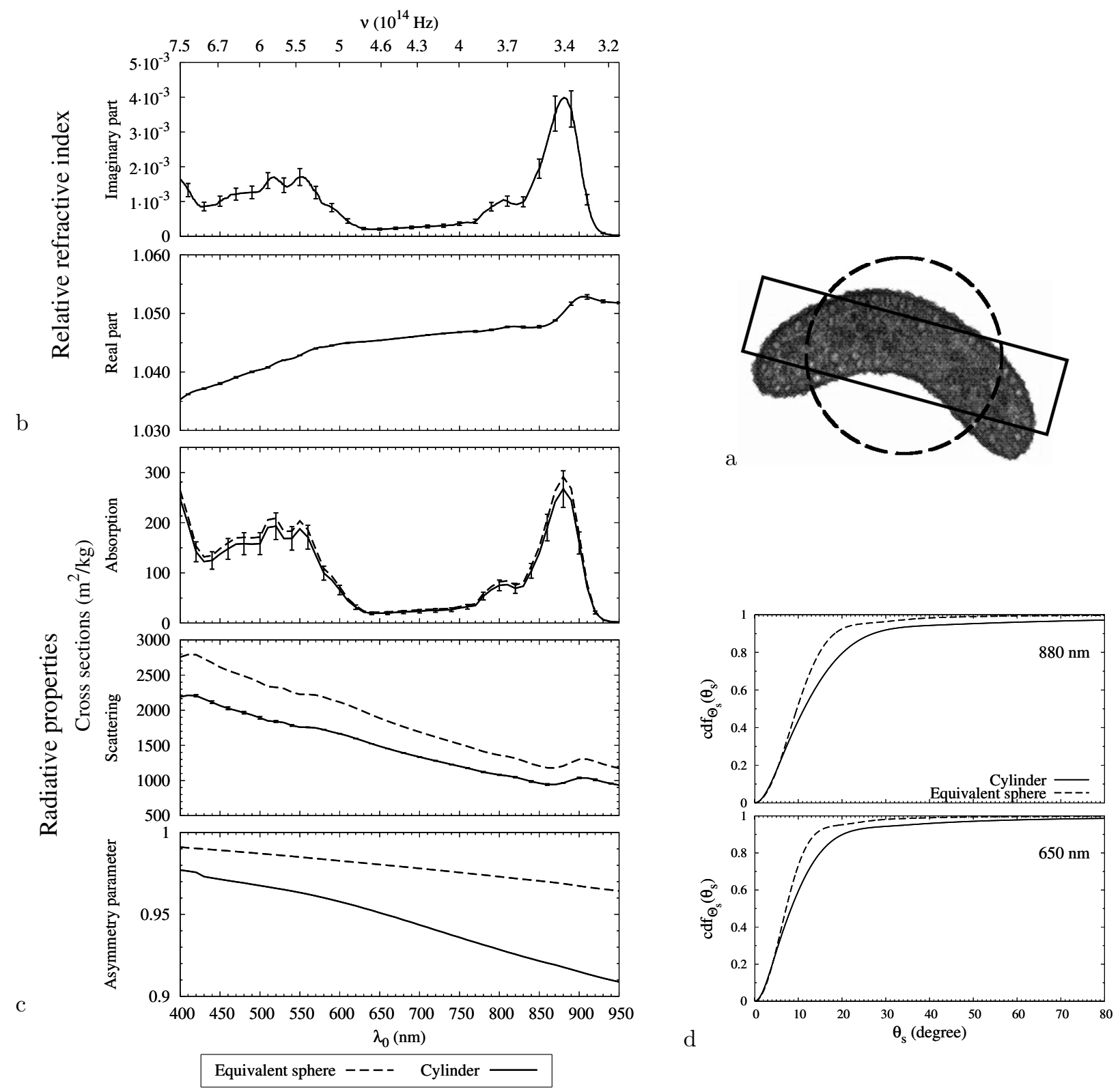

Figure 4: a: Rhodospirillum rubrum, a purple bacterium; cylindrical shape of aspect ratio $R=0.263$ (continuous line); size distribution $\bar{r}_{e q}=0.983 \mu \mathrm{m}$ and $s=1.1374$ (see Eq. 8) of the volume equivalent sphere (dashed line). b: Refractive index obtained for the following pigment composition (dry mass fractions $w_{\text {pig }, j}$ in \%): Bacteriochlorophyll a $1.75 \pm 0.25$, Specific carotenoids $0.35 \pm 0.05$; and the anchor point $n_{645 \mathrm{~nm}}=1.39$ derived from the following volume fractions: $f_{\text {wall }}=30 \%$, $f_{\text {cytoplasm }}=65 \%, f_{P H B}=5 \%$ (see Tab. 2); $x_{w}=0.82$. c: Radiative properties; $M_{\text {eff }}=1.1354 \cdot 10^{-15} \mathrm{~kg} \cdot$ particle ${ }^{-1}$. d: Cumulative phase function $c d f_{\Theta_{s}}\left(\theta_{s}\right)=2 \pi \int_{0}^{\theta_{s}} d \theta \sin (\theta) W_{s}(\theta) / \sigma_{s}$ at $\lambda_{0}=650$ and $880 \mathrm{~nm}$. 


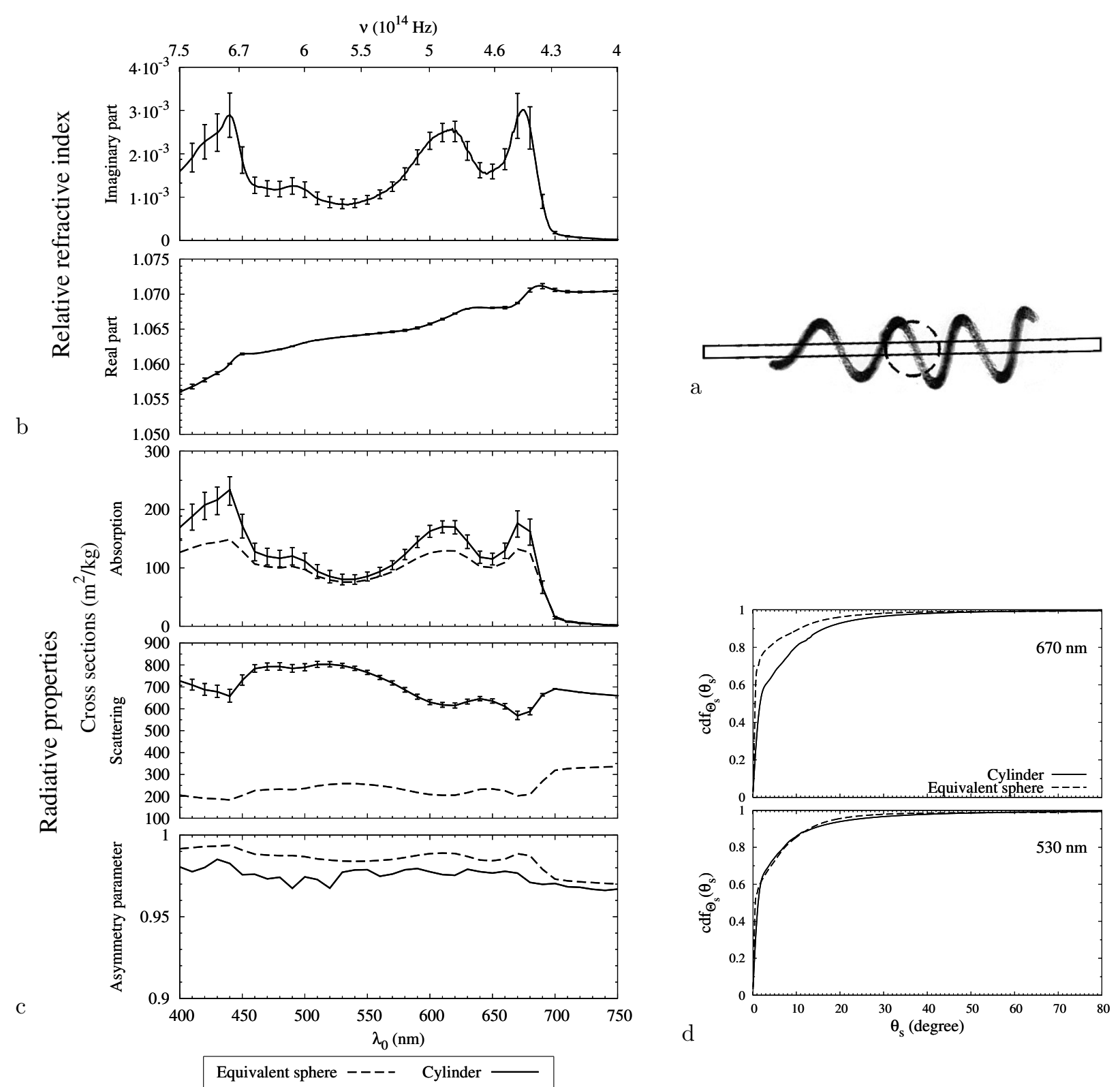

Figure 5: a: Arthrospira platensis, a multicellular filamentous cyanobacterium; cylindrical shape of aspect ratio $R=0.0284$ (continuous line); size distribution $\bar{r}_{e q}=15.52 \mu \mathrm{m}$ and $s=1.11$ (see Eq. 8) of the volume equivalent sphere (dashed line). b: Refractive index obtained for the following pigment composition (dry mass fractions $w_{p i g, j}$ in \%): Chlorophyll $a 1.0 \pm 0.2$, Phycocyanin C $9.0 \pm 0.6$, Allophycocyanin $6.0 \pm 0.4$, Photosynthetic carotenoids $0.17 \pm 0.03$, Photoprotective carotenoids $0.13 \pm 0.02$; and the anchor point $n_{825 \mathrm{~nm}}=1.42$ derived from the following volume fractions: $f_{\text {wall }}=10 \%, f_{\text {thylakoid }}=40 \%$, $f_{\text {nucleoid }}=5 \%, f_{\text {cytoplasm }}=45 \%$ (see Tab. 2$) ; x_{w}=0.8$. c: Radiative properties; $M_{\text {eff }}=4.7576 \cdot 10^{-12} \mathrm{~kg} \cdot$ particle ${ }^{-1}$. d: Cumulative phase function $c d f_{\Theta_{s}}\left(\theta_{s}\right)=2 \pi \int_{0}^{\theta_{s}} d \theta \sin (\theta) W_{s}(\theta) / \sigma_{s}$ at $\lambda_{0}=530$ and $670 \mathrm{~nm}$. 


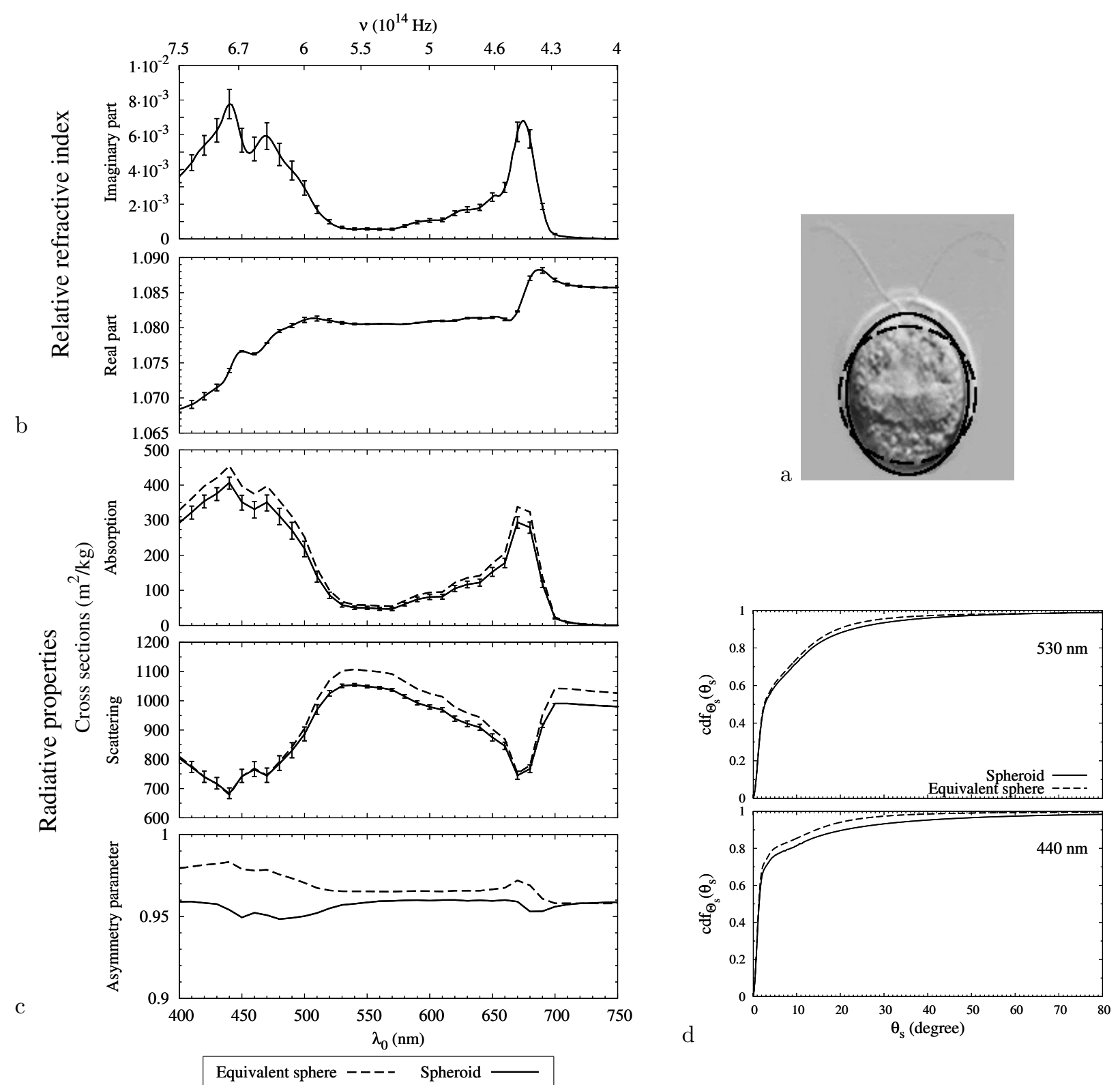

Figure 6: a: Chlamydomonas reinhardtii, an unicellular green alga; spheroidal shape of aspect ratio $R=0.837$ (continuous line); size distribution $\bar{r}_{e q}=3.963 \mu \mathrm{m}$ and $s=1.18$ (see Eq. 8) of the volume equivalent sphere (dashed line). b: Refractive index obtained for the following pigment composition (dry mass fractions $w_{p i g, j}$ in \%): Chlorophyll $a 2.2 \pm 0.2$, Chlorophyll $b$ $0.9 \pm 0.1$, Photosynthetic carotenoids $0.10 \pm 0.01$, Photoprotective carotenoids $0.60 \pm 0.09$; and the anchor point $n_{820} \mathrm{~nm}=1.44$ derived from the following volume fractions (from [99]): $f_{\text {wall }}=10 \%, f_{\text {nucleolus }}=1 \%, f_{\text {nucleus }}=7.5 \%, f_{\text {chloroplast }}=40 \%$, $f_{\text {mitochondria }}=3 \%, f_{\text {cytoplasm }}=38.5 \%$ (see Tab. 2); $x_{w}=0.78$. c: Radiative properties; $M_{\text {eff }}=9.8615 \cdot 10^{-14} \mathrm{~kg} \cdot$ particle ${ }^{-1}$. d: Cumulative phase function $c d f_{\Theta_{s}}\left(\theta_{s}\right)=2 \pi \int_{0}^{\theta_{s}} d \theta \sin (\theta) W_{s}(\theta) / \sigma_{s}$ at $\lambda_{0}=440$ and $530 \mathrm{~nm}$. 


\section{Validation of the predictive model from hemispherical transmittance spectra in multiple scattering configuration}

We hereafter focus on the last step of our methodological chain: the validation of the obtained radiative properties. This validation consists in the comparison between the normal-hemispherical transmittance spectra experimentally observed and those predicted by the resolution of the radiative transfer equation with the radiative properties obtained in Section 5 as input parameters.

\subsection{Experimental setup}

In this section, hemispherical transmittances have been measured with an optical bench specifically developed for this application. It is composed of a spectrofluorometer (SAFAS FLX, Monaco) equipped with a high-energy pulsed xenon lamp, and a high-accuracy integrating sphere of 6 inches that is coated with Spectralon (a patented highly-reflecting material, Labsphere, USA). Emitted light is guided from a monochromator to the integrating-sphere's sample port by using an optical fiber linked to optical devices generating a collimated incident beam. This bench enables to analyze transmittances in the range $2 \cdot 10^{-3}$ to 1 with an absolute tolerance of $0.2 \%$. The biomass concentrations $C_{x}$ and their standard deviations have been obtained by averaging 10 dry weight samples (oven, $110^{\circ} \mathrm{C}, 24$ hours). For each experiment and microorganism, the consistency of the obtained biomass concentration is analyzed as explained in Section 4.1.

\subsection{Monte Carlo resolution of the radiative transfer equation}

The predictive normal-hemispherical transmittance of a microorganism suspension with a given homogeneous concentration $C_{x}$ is hereafter calculated by rigorously solving the radiative transfer equation for the radiative properties obtained in Section 5 (the absorption coefficient is $K_{a, \nu}=C_{x} \sigma_{a, \nu}$, the scattering coefficient is $K_{s, \nu}=C_{x} \sigma_{s, \nu}$ and the differential scattering cross-section is $\left.W_{s, \nu}\right)$. The resolution makes use of the most recent advances in the field of radiative transfer Monte Carlo [100, 101, 102, 103], along the line of [12], including optical path sampling within non-gray absorbing and anisotropically scattering media with complex geometries. Rigorous estimation of the numerical error is also included: each estimation of the transmittance is calculated using $10^{7}$ samples, which corresponds to a standard deviation associated with the convergence of the Monte Carlo algorithm that is always inferior to $0.1 \%$ for the studied configurations ${ }^{8}$.

\subsection{Comparing model with experiments}

Experimental and predictive transmittance spectra obtained for the three studied microorganisms are respectively presented in Fig. 7, 8, and 9. Results are presented within the PAR: $\lambda_{0} \in[400 \mathrm{~nm}, 700 \mathrm{~nm}]$ for Arthrospira platensis and Chlamydomonas reinhardtii; $\lambda_{0} \in[400 \mathrm{~nm}, 850 \mathrm{~nm}]$ for Rhodospirillum rubrum (this bacteria exploits solar light up to $900 \mathrm{~nm}$ but our experimental setup is limited to $850 \mathrm{~nm}$ ). The errors associated with the experimental incertitude on the measure of the microorganism density $C_{x}$ and the pigment composition $w_{p i g, j}$ are hereafter investigated by reporting as errorbars the results of the model respectively obtained for the minimum and maximum values of these parameters, assuming monotony. The error due to the experimental measure of $C_{x}$ alone represents roughly $\frac{2}{3}$ of the total incertitude (i.e the sum of the incertitudes due to $C_{x}$ and $\left.w_{p i g, j}\right)$, which indicates that when performing radiative-transfer analysis, the incertitude on the pigment composition (which was identified as the main source of incertitude for the radiative properties) is no more predominant, and that the experimental efforts have to focus on the improvement of density-measurement accuracy.

When comparing experimental and predictive results, two situations are encountered. First, in the case of Chlamydomonas reinhardtii (Fig. 9), which has a shape that is very close to that of its chosen spheroidal description, in addition to a low elongation, both models (spheroid and equivalent-sphere) give similar

\footnotetext{
${ }^{8}$ We simulated radiative transfer both within an infinite slab and within the spectroscopy cuvette used for the experiments, including reflection and refraction at the suspension-quartz and quartz-air interfaces. The differences that we recorded between these two configurations (after baseline correction) are smaller than the accuracy of the experimental setup. Therefore, we present in Figs. 7, 8, 9, 10 and 11 the predictive hemispherical transmittance obtained for an infinite slab of microorganismsuspension with collimated normal incident-radiation on one side.
} 
and very accurate estimation of the transmittance, even for critical configurations with low absorption and high microorganisms concentration (e.g around $550 \mathrm{~nm}$ in Fig. 9.a). This result validates both the consistency of the whole methodological chain (the results are accurate), as well as the use of the Schiff approximation. Indeed, the tansmittances obtained with the Schiff approximation are very similar to those obtained with rigorous Lorenz-Mie calculation (the shape of the scatterer being almost spherical). In the other hand, for elongated microorganisms such as Arthrospira platensis (Fig. 8), the equivalent sphere approximation (or the criteria of equivalence) is clearly not valid: the sphere does not represent (in any way) the microorganism shape. Moreover, even if the description of Arthrospira platensis as a straight cylinder constitutes a better model when compared to the sphere, it still fails to give satisfactory predictions of the experimental results. The fact that we obtain a better accuracy for Chlamydomonas reinhardtii, which shape is very well described, motivates subsequent investigations of the opportunity to account for the helical shape of Arthrospira platensis (see Section 7).

Interestingly, the results obtained for Rhodospirillum rubrum appear as an intermediate case. Our modelled shape (a rod) is here relatively close to the actual shape of the microorganism, even if the slight curvature and the extremities are not accounted for. Consequently, the experimental data are well predicted over the covered wavelength-domain. Furthermore, the volume-equivalent sphere approximation is here rather acceptable. Nevertheless, a general discussion on the accuracy of equivalent-sphere can hardly be based on these results alone, since the multiple-scattering transmittances that we are here analyzing depend on transport properties (optical thickness, albedo of transport and asymmetry parameter) that can be surprisingly accurate or inaccurate depending on the compensation (as here) or addition of slight discrepancies in the radiative properties. In the present case (see Fig. 5) the increase of the scattering cross-section $\sigma_{s}$ (in comparison with cylinder) is compensated by the increase of the asymmetry parameter $g$, leading to an optical thickness $\sigma_{s}(1-g)$ that is only slightly modified. This point emphasizes the interest in other criteria than the scatterer-volume for the definition of equivalent-spheres, based on stronger physical basis [73]. It also emphasizes the limitations of the present multiple-scattering analysis and our interest in further validations based on single-scattering experiments (see Sec. 7).

To sum up, the present analysis of normal-hemispherical transmittances of three different structural class of photosynthetic microorganisms demonstrates that accounting for the scatterer-shape, even if the actual shape is only approached, always leads to an improvement of the radiative-transfer description. Very good theoretical predictions are indeed obtained when the shape of the scatterer is properly described (e.g for Chlamydomonas reinhardtii and for Rhodospirillum rubrum), whereas a rough approximation (e.g for Arthrospira platensis) leads to significant deviations. In such cases, the assessment of radiation-field that is necessary to properly formulate kinetic and energetic coupling within photobioreactors is not satisfactory, regardless of the technique used for solving the radiative transfer equation: the use of advanced radiativetransfer resolution techniques (as here or in [12]) is only pertinent if radiative properties are accurately known. 

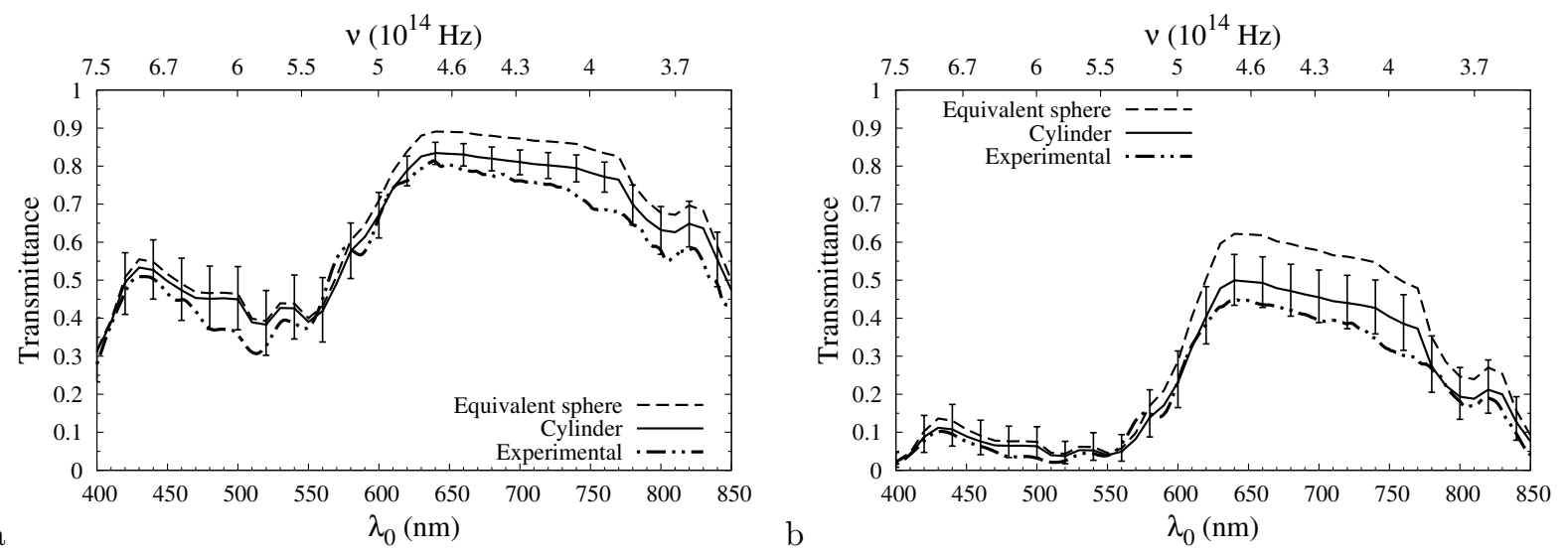

Figure 7: Hemispherical transmittance spectra of Rhodospirillum rubrum for a cuvette of thickness $1 \mathrm{~cm}$. a: $C_{x}=0.41 \pm$ 0.04 g. $L^{-1}$. b: $C_{x}=1.2 \pm 0.1$ g. $L^{-1}$.

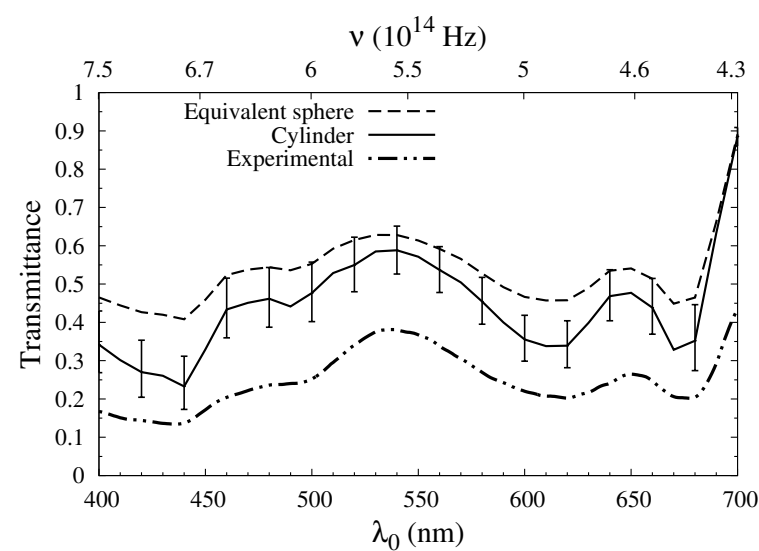

Figure 8: Hemispherical transmittance spectrum of Arthrospira platensis for a cuvette of thickness $1 \mathrm{~cm}$ and $C_{x}=0.60 \pm$ 0.06 g. $L^{-1}$ 


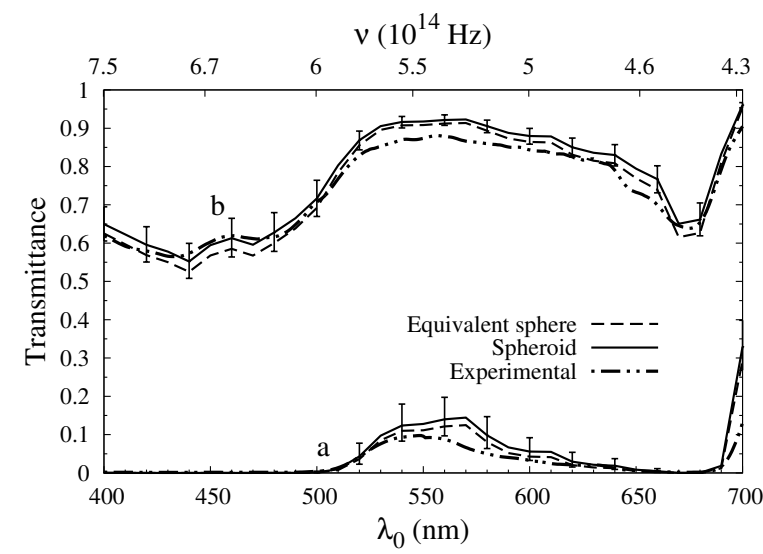

Figure 9: Hemispherical transmittance spectra of Chlamydomonas reinhardtii for a cuvette of thickness $1 \mathrm{~cm}$. a: $C_{x}=$ $2.3 \pm 0.2$ g. $L^{-1}$. b: $C_{x}=0.14 \pm 0.02 g . L^{-1}$ 


\subsection{Analysis of the anchor point from a simple inversion method}

Here, our model is further put to the test through an investigation at the anchoring frequency alone (where the microorganisms do not absorb radiation) in order to analyze the influence of the anchor point and validate its predictive determination.

All the complexity associated with the microorganism heterogeneities is contained in the value $n_{\nu_{p}}$ of the anchor point. Yet, this anchoring is predicted from data upon which we place limited confidence (see Tab. 2). Therefore, it is legitimate to question the causes behind the discrepancies observed in the case of Arthrospira platensis in Fig. 5: is the poor description of the microorganism shape to be incriminated, or a poor estimation of $n_{\nu_{p}}$ ? For that purpose, we hereafter perform a simple inversion consisting in the determination of the experimental value $n_{\nu_{p}}^{e x p}$ of the anchor point insuring that measured transmittance $T_{\nu_{p}}^{e x p}$ and predictive transmittance $T_{\nu_{p}}\left(n_{\nu_{p}}\right)$ are equal. This inversion is relatively simple because, first, only one parameter is inverted, second, it is only performed for one microorganism density $C_{x}$ and one frequency $\nu_{p}$, which implies that the criteria of equivalence is simply the equality $T_{\nu_{p}}\left(n_{\nu_{p}}^{e x p}\right)=T_{\nu_{p}}^{e x p}$. Moreover, the microorganisms being non-absorbing at the frequency $\nu_{p}$, the present inversion is independent of our database of pigment absorption. Additionally, we consider that the refractive index of microorganisms is undoubtedly contained between 1.33 (water) and 1.7 (extremely high value in itself) and only this range is explored. This inversion is performed for Arthrospira platensis in Fig. 10 and for Chlamydomonas reinhardtii in Fig. 11, where the same uncertainty of measurement as in Section 6.3 are reported.

For Chlamydomonas reinhardtii the inversion gives $n_{\nu_{p}}^{e x p}=1.44 \pm 0.01$, which is fully consistent with the value previously predicted. For Arthrospira platensis, in the other hand, there is no refractive index $n_{\nu_{p}}$ reconciling experiments and simulations within the range $n_{\nu_{p}} \in[1.33,1.7]$ : there is no solution to the inverse problem. Therefore, the value of the anchor point used in Section 6.3 is not responsible for the unsatisfactory results of Fig 8 because, regardless of the $n_{\nu_{p}}$ value (within a coherent range), the model does not allow to describe experimental results. The fact that the inversion gives coherent results in the case of Chlamydomonas reinhardtii, whose shape is accurately described in our model, and that it fails in the case of Arthrospira platensis, confirms the importance of using an accurate shape-model. Moreover, we record that for the equivalent-sphere model of Arthrospira platensis the inversion is far to be achieved, while for the straight-cylinder model, although the inversion is not possible, $T_{\nu_{p}}\left(n_{\nu_{p}}\right)$ approaches the experimental value $T_{\nu_{p}}^{e x p}$. This result constitutes a further motivation for a subsequent investigation of the helical shape of Arthrospira platensis. 


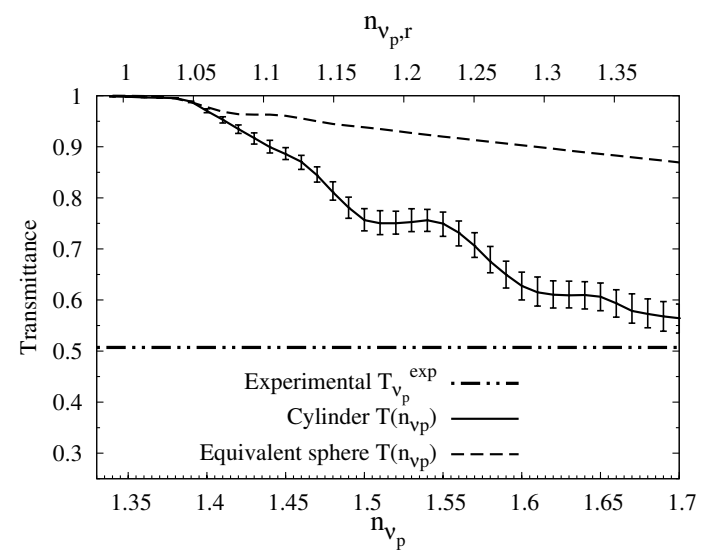

Figure 10: Hemispherical transmittance of Arthrospira platensis at $\lambda_{0}=825 \mathrm{~nm}$, for a cuvette of thickness $1 \mathrm{~cm}$ and $C_{x}=$ $1.1 \pm 0.1 \mathrm{~g} \cdot \mathrm{L}^{-1}$. No inversion is found within the range $n_{\nu_{p}} \in[1.33,1.7]$.

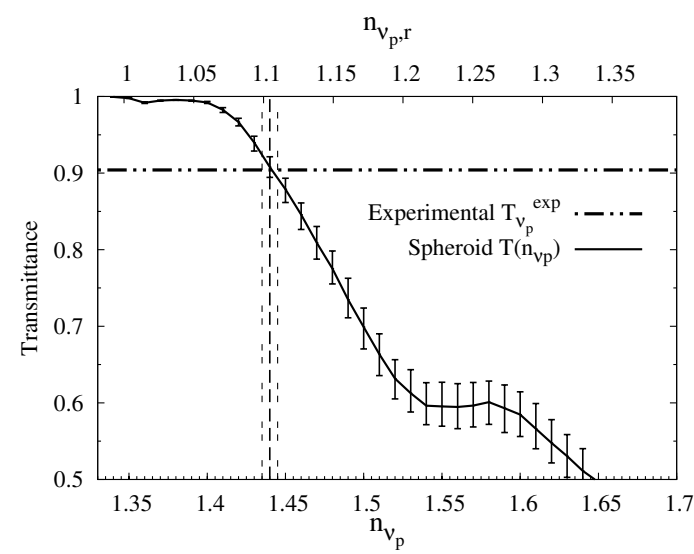

Figure 11: Hemispherical transmittance of Chlamydomonas reinhardtii at $\lambda_{0}=820 \mathrm{~nm}$, for a cuvette of thickness $1 \mathrm{~cm}$ and $C_{x}=1.0 \pm 0.1 \mathrm{~g} \cdot \mathrm{L}^{-1}$. The anchor point $n_{\nu_{p}}^{e x p}$ obtained by inversion is $1.44 \pm 0.01$. 


\section{Conclusion and perspectives}

The present article is the result of ten years of modelling and experimenting with light-scattering by microalgae. It is presented here both as a theoretical predictive-methodology and as an overview of techniques for computing optical and radiative properties of photosynthetic microorganisms. The calculation of such properties is required for various applications, especially in the radiative-transfer analysis of photobioreactors, where predictive calculation appears as an interesting complement to experimental assessment, because of the strong variations of microorganism features as a function of culture conditions (light-frequency, radiation field, mineral or $\mathrm{CO}_{2}$ limitations, starvations, reactor geometry, time varying solar illumination, etc). In this regard, the methodological chain that has been presented aims at fully meeting the needs of photobioreactor study: its input parameters are precisely the most sensitive among the microorganisms' features (photosynthetic-pigment content, geometry, intracellular water content) and its genericity allows us to treat, with the same procedure, representatives of the three main classes of photosynthetic microorganisms i.e photosynthetic bacteria, cyanobacteria and eukaryotic microalgae, which illustrate our ability to encompass the diversity of the microbial world.

A crucial difficulty encountered by this achievement is that the size parameters and elongations of photosynthetic microorganisms are, at present, out of the range of accuracy or capacity of analytical and numerical rigorous electromagnetic-codes (e.g. respectively in the case of T-matrix or FDTD methods) and also outside the range of validity of the standard Rayleigh or geometrical optics approximation. In this context, the use of the Schiff approximation has been proven to be particularly well-suited to the computation of the cross-sections and phase functions, without any conceptual difficulty in the treatment of microorganisms with complex shapes. In this first paper however, the authors intentionally decided to restrict their choice to microorganisms having shapes which remain very close to spheroids or cylinders, for which theoretical solutions have already been derived in the associated literature (except for the phase functions). Results involving advanced geometries will be reported elsewhere.

A comparison of predictive calculations with experiments, analysing the hemispherical transmittance of microorganism suspensions, establishes that the proposed methodological chain (optical properties calculated from in vivo pigment databases and the Kramers-Krönig relations, combined with the Schiff approximation for the radiative properties evaluation) leads to accurate calculations when the model-shape is adequate and the input parameters are known with confidence (size distribution, intracellular volume fractions of organelles, as well as pigment and water content). Among these parameters, the volume fractions of organelles are the most difficult to access, but the results on Rhodospirillum rubrum (for which the shape was accurately described as a cylinder) indicate that a rough estimation from optical micrographs suffices to obtain a fair evaluation of the radiative properties. These organelles' volume-fractions are used to determine the real part of the refractive index of the microorganisms, which is a delicate part of the model. Therefore, the consistency of these results has been reinforced by a dedicated validation consisting in an inversion from transmittance measurement at a non-absorbed wavelength (case study with spheroidal Chlamydomonas reinhardtii cells). Overall, we retain that the calculated radiative properties were always more pertinent when the shape of the microorganisms was accurately accounted for (thanks to the Schiff approximation), in comparison with the volume-equivalent sphere approximation (using rigorous Lorenz-Mie resolution). It has even been proved, in the case of the elongated microorganism Arthrospira platensis, that no volume-equivalent sphere exists which gives concordance with the experimental measurements.

Although the presented methodological chain is shown to be promising, its weaknesses have been rigorously evidenced and discussed, together with a sensitivity analysis of some important input parameters (pigment content, biomass concentration). This should allow for the discussion of interesting perspectives involving different scientific communities, through the identification of some important experimental and theoretical points that will have to be significantly improved in the future.

First, the measurement of the in vivo absorption-properties of pigment still require the continuation of the research effort that was engaged during the seventies, when different scientific communities identified that any calculation of the radiative properties of photosynthetic-cells should rely on this information. Here we have revisited the pioneering work of Bidigare et al. on eukaryotic algae [41] and along these lines, we have performed our own measurements on cyanobacteria and bacteria, leading to the database provided in 
the supplementary material [89], that gathers in vivo properties of fourteen photosynthetic-pigments which are among the most important in nature. Although quite extensive, this database is far to be exhaustive and further work will be necessary in order to include numerous other pigments, in particular many carotenoids that have so far been treated as a photoprotective and a photosynthetic mixture. This task is far from trivial because each new pigment requires a complete theoretical and experimental methodology, as explained in the core of this article, as well as a careful validation procedure, the calculation of the radiative properties being extremely sensitive to the resulting absorption-spectra.

Second, it must be emphasized that the values currently obtained for the real part of the refractive indices are to date limited by the lack of information and confidence concerning the refractive indices and volume fractions of the different internal structures of photosynthetic microorganisms (that are used within the Bruggemann mixing rule). Different techniques must be envisaged in the future in order to measure them with better accuracy. An additional aspect that requires improvement is the experimental accuracy of the pigment-content measurement (involved in the calculation of the imaginary part of refractive indices) which is the main source of uncertainty regarding radiative properties.

Another significant challenge for future work is the implementation of the Schiff approximation in complex geometries for which the intersection of incident rays with the scatterer cannot be calculated analytically (unlike the calculations for spheroids and cylinders that have been presented here). Particularly interesting complex-geometries are, for instance, rotationally-symmetric shapes [104] such as Tchebytchev particles [105, 106] and superspheroids [107], as well as the helical shape of Arthrospira platensis.

Finally, considering the complexity of shapes, the size parameter range and the refractive indices of photosynthetic microorganisms, it seems evident that the Schiff approximation should retain increasing attention and consideration for a long time, even if concurrent exact solutions and numerical methods are continuously in progress. The capabilities and limitations of the Schiff approximation are therefore currently under active investigation, in particular thanks to its comparison with experimental measurements in single scattering condition [8]. The microwave analog measurements practised by the electromagnetic community [17] are equally promising developments.

\section{Acknowledgements}

This work has been sponsored by the French government research-program "Investissements d'avenir" through the ANR programs PHOTOBIOH2 (2005-08), BIOSOLIS (2008-11), ALGOH2 (2011-15), PRIAM (2013-15), the IMobS3 Laboratory of Excellence (ANR-10-LABX-16-01), by the European Union through the program "Regional competitiveness and employment" 2007-2013 (ERDF Auvergne region), and by the Auvergne region. It is also founded by the CNRS through the PIE program PHOTORAD (2010-11) and the PEPS program "Intensification des transferts radiatifs pour le developpement de photobioreacteurs a haute productivite volumique" (2012-13).

\section{References}

[1] S. Aiba, Growth kinetics of photosynthetic micro-organisms, Adv. Biochem. Eng. 23 (1982) 85-156.

[2] J. F. Cornet, C. G. Dussap, G. Dubertret, A structured model for simulation of cultures of the cyanobacterium spirulina platensis in photobioreactors: I. coupling between light transfer and growth kinetics, Biotechnology and Bioengineering 40 (7) (1992) 817-825.

[3] J.-F. Cornet, C. Dussap, J.-B. Gros, Kinetics and energetics of photosynthetic micro-organisms in photobioreactors, in: Bioprocess and Algae Reactor Technology, Apoptosis, Vol. 59 of Advances in Biochemical Engineering Biotechnology, Springer Berlin Heidelberg, 1998, pp. 153-224.

[4] J. F. Cornet, C. G. Dussap, A simple and reliable formula for assessment of maximum volumetric productivities in photobioreactors, Biotechnology Progress 25 (2) (2009) 424-435.

[5] J. F. Cornet, Calculation of optimal design and ideal productivities of volumetrically lightened photobioreactors using the constructal approach, Chemical Engineering Science 65 (2) (2010) 985-998.

[6] J. Pruvost, J. F. Cornet, Knowledge models for the engineering and optimization of photobioreactors, C. Posten, C. Walter (Eds.), Microalgal Biotechnology: Potential and Production, De Gruyter, Berlin, Germany, 2012.

[7] H. Takache, G. Christophe, J.-F. Cornet, J. Pruvost, Experimental and theoretical assessment of maximum productivities for the microalgae chlamydomonas reinhardtii in two different geometries of photobioreactors, Biotechnology Progress 26 (2) (2010) 431-440. 
[8] L. Pilon, H. Berberoglu, R. Kandilian, Radiation transfer in photobiological carbon dioxide fixation and fuel production by microalgae, Journal of Quantitative Spectroscopy and Radiative Transfer 112 (17) (2011) 2639-2660.

[9] A. E. Cassano, C. A. Martin, R. J. Brandi, O. M. Alfano, Photoreactor analysis and design : fundamentals and applications, Ind. Eng. Chem. Res. 34 (1995) 2155-2201.

[10] Z. Csogor, M. Herrenbauer, K. Schmidt, C. Posten, Light distribution in a novel photobioreactor - modelling for optimization, Journal of Applied Phycology 13 (2001) 325-333.

[11] L. Pottier, J. Pruvost, J. Deremetz, J. F. Cornet, J. Legrand, C. G. Dussap, A fully predictive model for one-dimensional light attenuation by chlamydomonas reinhardtii in a torus photobioreactor, Biotechnol. Bioeng. 91 (5) (2005) 569-582.

[12] J. Dauchet, S. Blanco, J.-F. Cornet, M. El Hafi, V. Eymet, R. Fournier, The practice of recent radiative transfer monte carlo advances and its contribution to the field of microorganisms cultivation in photobioreactors, Journal of Quantitative Spectroscopy and Radiative Transfer 128 (0) (2013) 52-59.

[13] M. P. Mengüç, Characterization of fine particles via elliptically-polarized light scattering, Purdue Heat Transfer Celebration (2002)

[14] O. Muoz, J. W. Hovenier, Laboratory measurements of single light scattering by ensembles of randomly oriented small irregular particles in air. a review, Journal of Quantitative Spectroscopy and Radiative Transfer 112 (11) (2011) 16461657.

[15] H. Berberoglu, L. Pilon, A. Melis, Radiation characteristics of chlamydomonas reinhardtii cc125 and its truncated chlorophyll antenna transformants tla1, tlax and tla1-cw+, International Journal of Hydrogen Energy 33 (22) (2008) 6467-6483.

[16] H. Berberoglu, P. S. Gomez, L. Pilon, Radiation characteristics of botryococcus braunii, chlorococcum littorale, and chlorella sp. used for co2 fixation and biofuel production, Journal of Quantitative Spectroscopy and Radiative Transfer 110 (17) (2009) 1879-1893.

[17] R. Vaillon, J. M. Geffrin, C. Eyraud, O. Merchiers, P. Sabouroux, B. Lacroix, A novel implementation of a microwave analog to light scattering measurement set-up, Journal of Quantitative Spectroscopy and Radiative Transfer 112 (11) (2011) 1753-1760.

[18] T. Wriedt, Light scattering theories and computer codes, Journal of Quantitative Spectroscopy and Radiative Transfer 110 (11) (2009) 833-843.

[19] F. M. Kahnert, Numerical methods in electromagnetic scattering theory, Journal of Quantitative Spectroscopy and Radiative Transfer 7980 (0) (2003) 775-824.

[20] B. Fernandes, G. Dragone, J. Teixeira, A. Vicente, Light regime characterization in an airlift photobioreactor for production of microalgae with high starch content, Applied Biochemistry and Biotechnology 161 (2010) $218-226$.

[21] E. Franco-Lara, J. Havel, F. Peterat, D. Weuster-Botz, Model-supported optimization of phototrophic growth in a stirred-tank photobioreactor, Biotechnology and Bioengineering 95 (6) (2006) 1177-1187.

[22] I. S. Suh, S. B. Lee, A light distribution model for an internally radiating photobioreactor, Biotechnology and Bioengineering 82 (2) (2003) 180-189.

[23] I. S. Suh, S. B. Lee, Optimization of radiator position in an internally radiating photobioreactor: A model simulation study, J. Microbiol. Biotechnol. 13 (2003) 789-793.

[24] I. S. Suh, S. B. Lee, Cultivation of a cyanobacterium in an internally radiating air-lift photobioreactor, Journal of Applied Phycology 13 (2001) 381-388.

[25] W. W. Su, J. Li, N. S. Xu, State and parameter estimation of microalgal photobioreactor cultures based on local irradiance measurement, Journal of Biotechnology 105 (1-2) (2003) 165-178.

[26] J. F. Cornet, C. G. Dussap, P. Cluzel, G. Dubertret, A structured model for simulation of cultures of the cyanobacterium spirulina platensis in photobioreactors: Ii. identification of kinetic parameters under light and mineral limitations, Biotechnology and Bioengineering 40 (7) (1992) 826-834.

[27] B. Farges, C. Laroche, J. F. Cornet, C. G. Dussap, Spectral kinetic modeling and long-term behavior assessment of arthrospira platensis growth in photobioreactor under red (620 nm) light illumination, Biotechnology Progress 25 (1) (2009) 151-162.

[28] H. C. van de Hulst, Light scattering by small particules, Dover Publication, Inc., 1981.

[29] C. F. Bohren, D. R. Huffman, Absorption and scattering of light by small particles, New York, Wiley-Interscience, 1983.

[30] M. I. Mishchenko, J. W. Hovenier, L. D. Travis, Light scattering by nonspherical particles: theory, measurements, and applications, Academic Press, 2000.

[31] M. Mishchenko, L. Travis, A. Lacis, Scattering, absorption, and emission of light by small particles, Cambridge Univ Pr, 2002, The Lorenz-Mie code used in the present article is available at www.giss.nasa.gov/staff/mmishchenko.

[32] S. Bernard, T. A. Probyn, A. Quirantes, Simulating the optical properties of phytoplankton cells using a two-layered spherical geometry, Biogeosciences Discussions 6 (1) (2009) 1497-1563.

[33] T. T. Wu, J. Y. Qu, M. Xu, Unified mie and fractal scattering by biological cells and subcellular structures, Optics Letters 32 (16) (2007) 2324-2326.

[34] W. Choi, C.-C. Yu, C. Fang-Yen, K. Badizadegan, R. Dasari, M. S. Feld, Field-based angle-resolved light-scattering study of single live cells, Optics Letters 33 (14) (2008) 1596-1598.

[35] L. Kolokolova, B. A. S. Gustafsonm, Scattering by inhomogeneous particles: microwave analog experiments and comparison to effective medium theories, Journal of Quantitative Spectroscopy and Radiative Transfer 70 (4-6) (2001) 611-625.

[36] J. Dauchet, Analyse radiative des photobioréacteurs, Ph.D. thesis, University Blaise Pascal - Clermont II, The corresponding publication is being prepared. (2012).

[37] Z. P. Wang, Y. Zhao, Morphological reversion of spirulina (arthrospira) platensis (cyanophyta): from linear to helical, Journal of Phycology 41 (3) (2005) 622-628. 
[38] N. Hoepffner, S. Sathyendranath, Effect of pigment composition on absorption properties of phytoplankton, Marine Ecology Progress Series 73 (1) (1991) 11-23.

[39] A. Morel, A. Bricaud, Theoretical results concerning light absorption in a discrete medium, and application to specific absorption of phytoplankton, Deep Sea Research Part A. Oceanographic Research Papers 28 (11) (1981) 1375-1393.

[40] A. Bricaud, A. Morel, Light attenuation and scattering by phytoplanktonic cells: a theoretical modeling, Appl. Opt. 25 (4) (1986) 571-580.

[41] R. R. Bidigare, M. E. Ondrusek, J. H. Morrow, D. A. Kiefer, In-vivo absorption properties of algal pigments, Vol. 1302, SPIE, 1990, pp. 290-302.

[42] B. Wozniak, J. Dera, D. Ficek, R. Majchrowski, S. Kaczmarek, M. Ostrowska, O. I. Koblentz-Mishke, Model of the in vivo spectral absorption of algal pigments. part 1. mathematical apparatus, Oceanologia 42 (2) (2000) $177-190$.

[43] E. Aas, Refractive index of phytoplankton derived from its metabolite composition, Journal of Plankton Research 18 (12) (1996) 2223-2249.

[44] P. J. Wyatt, Differential light scattering: a physical method for identifying living bacterial cells, Applied Optics 7 (10) (1968) 1879-1896.

[45] P. J. Wyatt, Cell wall thickness, size distribution, refractive index ratio and dry weight content of living bacteria (stapylococcus aureus), Nature 226 (5242) (1970) 277-279.

[46] P. J. Wyatt, Light scattering in the microbial world, Journal of Colloid and Interface Science 39 (3) (1972) $479-491$.

[47] V. Tychinsky, A. Tikhonov, Interference microscopy in cell biophysics. 1. principles and methodological aspects of coherent phase microscopy, Cell Biochemistry and Biophysics 58 (2010) 107-116.

[48] V. Tychinsky, A. Tikhonov, Interference microscopy in cell biophysics. 2. visualization of individual cells and energytransducing organelles, Cell Biochemistry and Biophysics 58 (2010) 117-128.

[49] V. Tychinsky, The metabolic component of cellular refractivity and its importance for optical cytometry, Journal of Biophotonics 2 (8-9) (2009) 494-504.

[50] N. Lue, G. Popescu, T. Ikeda, R. R. Dasari, K. Badizadegan, M. S. Feld, Live cell refractometry using microfluidic devices, Optics Letters 31 (18) (2006) 2759-2761.

[51] W. Choi, C. Fang-Yen, K. Badizadegan, S. Oh, N. Lue, R. R. Dasari, M. S. Feld, Tomographic phase microscopy, Nat Meth 4 (9) (2007) 717-719.

[52] Y. Park, M. Diez-Silva, G. Popescu, G. Lykotrafitis, W. Choi, M. S. Feld, S. Suresh, Refractive index maps and membrane dynamics of human red blood cells parasitized by plasmodium falciparum, Proceedings of the National Academy of Sciences 105 (37) (2008) 13730-13735.

[53] A. Loparev, A. Kretushev, V. Tychinsky, Coherent phase microscopy: a new method of identification of intracellular structures based on their optical and morphometric parameters, Biofizika 53 (2) (2008) 299-304.

[54] R. Cisek, L. Spencer, N. Prent, D. Zigmantas, G. Espie, V. Barzda, Optical microscopy in photosynthesis, Photosynthesis Research 102 (2009) 111-141.

[55] Virtual chemistry laboratory, University of Oxford, http://www.chem.ox.ac.uk/vrchemistry.

[56] E. H. Harris, The Chlamydomonas Sourcebook: Introduction to Chlamydomonas and its laboratory use. Second ed, Academic Press: San Diego, California, USA, 2009.

[57] P. S. Tuminello, E. T. Arakawa, B. N. Khare, J. M. Wrobel, M. R. Querry, M. E. Milham, Optical properties of bacillus subtilis spores from 0.2 to $2.5 \nu \mathrm{m}$, Applied Optics 36 (13) (1997) 2818-2824.

[58] J. M. Perrin, P. L. Lamy, On the validity of effective-medium theories in the case of light extinction by inhomogeneous dust particles, Astrophysical Journal 364 (1990) 146-151.

[59] N. V. Voshchinnikov, G. Videen, T. Henning, Effective medium theories for irregular fluffy structures: aggregation of small particles, Appl. Opt. 46 (19) (2007) 4065-4072.

[60] C. F. Bohren, L. J. Battan, Radar backscattering by inhomogeneous precipitation particles, Journal of the Atmospheric Sciences 37 (8) (1980) 1821-1827.

[61] C. Erlick, Effective refractive indices of water and sulfate drops containing absorbing inclusions, Journal of the Atmospheric Sciences 63 (2) (2006) 754-763.

[62] A. Worringen, M. Ebert, T. Trautmann, S. Weinbruch, G. Helas, Optical properties of internally mixed ammonium sulfate and soot particles - a study of individual aerosol particles and ambient aerosol populations, Appl. Opt. 47 (21) (2008) 3835-3845.

[63] A. H. Sihvola, Electromagnetic mixing formulas and applications, IEE, London, 1999.

[64] S. Bernard, T. A. Probyn, R. G. Barlow, Measured and modelled optical properties of particulate matter in the southern benguela, South African Journal of Science 97 (9-10) (2001) 410-420.

[65] N. G. Van Kampen, F. Lurçat, Causalité et relations de kramers-kronig, J. Phys. Radium 22 (3) (1961) 179-191.

[66] J. S. Toll, Causality and the dispersion relation: Logical foundations, Physical Review 104 (6) (1956) 1760-1770.

[67] V. Lucarini, Kramers-Kronig relations in optical materials research, Springer, 2005.

[68] K. R. Naqvi, M. N. Merzlyak, T. B. Melo, Absorption and scattering of light by suspensions of cells and subcellular particles: an analysis in terms of kramers-kronig relations, Photochem. Photobiol. Sci. 3 (2004) $132-137$.

[69] D. De Sousa Meneses, B. Rousseau, P. Echegut, P. Simon, Retrieval of linear optical functions from finite range spectra, Appl. Spectrosc. 61 (12) (2007) 1390-1397.

[70] J. A. Roels, Kinetics and energetics in biotechnology, Elsevier Biomedical Press, Amsterdam, 1983.

[71] P. Stier, J. H. Seinfeld, S. Kinne, O. Boucher, Aerosol absorption and radiative forcing, Atmospheric Chemistry and Physics 7 (3) (2007) 7171-7233.

[72] O. Schmid, D. Chand, E. Karg, P. Guyon, G. P. Frank, E. Swietlicki, M. O. Andreae, Derivation of the density and refractive index of organic matter and elemental carbon from closure between physical and chemical aerosol properties, 
Environmental Science \& Technology 43 (4) (2009) 1166-1172.

[73] P. Yang, Z. Zhang, B. A. Baum, H.-L. Huang, Y. Hu, A new look at anomalous diffraction theory (adt): Algorithm in cumulative projected-area distribution domain and modified adt, Journal of Quantitative Spectroscopy and Radiative Transfer 89 (1-4) (2004) 421-442.

[74] T. Nousiainen, Optical modeling of mineral dust particles: A review, Journal of Quantitative Spectroscopy and Radiative Transfer 110 (14-16) (2009) 1261-1279.

[75] L. Liu, M. I. Mishchenko, W. P. Arnott, A study of radiative properties of fractal soot aggregates using the superposition T-matrix method, Journal of Quantitative Spectroscopy and Radiative Transfer 109 (15) (2008) 2656 - 2663.

[76] A. J. Baran, A review of the light scattering properties of cirrus, Journal of Quantitative Spectroscopy and Radiative Transfer 110 (14-16) (2009) 1239-1260.

[77] T. C. Bond, R. W. Bergstrom, Light absorption by carbonaceous particles: An investigative review, Aerosol Science and Technology 40 (1) (2006) 27-67.

[78] C. M. Sorensen, Light scattering by fractal aggregates: A review, Aerosol Science and Technology 35 (2) (2001) $648-687$.

[79] S. Asano, M. Sato, Light scattering by randomly oriented spheroidal particles, Applied Optics 19 (6) (1980) 962-974.

[80] J. Hellmers, V. Schmidt, T. Wriedt, Improving the numerical stability of T-matrix light scattering calculations for extreme particle shapes using the nullfield method with discrete sources, Journal of Quantitative Spectroscopy and Radiative Transfer 112 (11) (2011) 1679-1686.

[81] L. I. Schiff, Approximation method for high-energy potential scattering, Physical Review 103 (2) (1956) 443-453.

[82] M. Xu, A. Katz, Statistical interpretation of light anomalous diffraction by small particles and its applications in bio-agent detection and monitoring, in: Light Scattering Reviews 3, Springer Praxis Books, 2008, pp. 27-67.

[83] J. Q. Zhao, Y. Q. Hu, Bridging technique for calculating the extinction efficiency of arbitrary shaped particles, Appl. Opt. $42(24)$ (2003) 4937-4945.

[84] J. Q. Zhao, G. Shi, H. Che, G. Cheng, Approximations of the scattering phase functions of particles, Advances in Atmospheric Sciences 23 (2006) 802-808.

[85] W. A. Farone, M. J. I. Robinson, The range of validity of the anomalous diffraction approximation to electromagnetic scattering by a sphere, Appl. Opt. 7 (4) (1968) 643-645.

[86] Y. Liu, W. P. Arnott, J. Hallett, Anomalous diffraction theory for arbitrarily oriented finite circular cylinders and comparison with exact T-matrix results, Appl. Opt. 37 (21) (1998) 5019-5030.

[87] B. F. Dudley, B. A. Steiber, P. Latimer, Absolute optical cross sections of cells and chloroplasts, Archives of biochemistry and biophysics 135 (1969) 79-108.

[88] T. Pullerits, V. Sundstrm, Photosynthetic light-harvesting pigment-protein complexes: Toward understanding how and why, Accounts of Chemical Research 29 (8) (1996) 381-389.

[89] StarWest, Edstar development environment project, http://edstar.lmd.jussieu.fr/databases.

[90] J. Myers, K. A. Kratz, Relation between pigment content and photosynthetic characteristics in blue green alga, J. Gen. Physiol. 39 (1955) 11-22.

[91] A. Bennet, L. Bogorad, Complementary chromatic adaptation in a filamentous blue-green alga, J. Cell Biol. 58 (1973) 419-435.

[92] J. D. H. Strickland, T. R. Parsons, A practical handbook of seawater analysis: Pigment analysis, Bulletin of Fisheries Research Board of Canada 167.

[93] V. L. P., G. A. F., Pigment-protein complexes derived from Rhodospirillum rubrum chromatophores by enzymatic digestion, Biochim. Biophys. Acta 143 (1967) 144-153.

[94] B. G., D. I., Bacterial dry matter content and biomass estimations, Applied and environmental microbiology 48 (1984) $755-757$.

[95] K. Inoue, M. Nishimura, B. B. Nayak, K. Kogure, Separation of marine bacteria according to buoyant density by use of the density-dependent cell sorting method, Applied and environmental microbiology 73 (2007) 1049-1053.

[96] K. F. A. Ross, E. Billing, The water and solid content of living bacterial spores and vegetative cells as indicated by refractive index measurements, Journal of General Microbiology 16 (2) (1957) 418-425.

[97] I. Thormählen, J. Straub, U. Grigull, Refractive index of water and its dependence on wavelength, temperature, and density, Journal of Physical and Chemical Reference Data 14 (1985) 933-945.

[98] G. Lambeek, E. Vorenkamp, A. J. Schouten, Structural study of langmuir-blodgett mono- and multilayers of poly(btahydroxybutyrate), Macromolecules 28 (1995) 2023-2032.

[99] F. Schtz, H. Bathelt, C. G. Arnold, O. Schimmer, Die architektur und organisation der chlamydomonas-zelle, Protoplasma 75 (1972) 229-254.

[100] J. R. Howell, The monte carlo method in radiative heat transfer, Journal of Heat Transfer 120 (3) (1998) 547-560.

[101] M. F. Modest, Radiative Heat Transfer Second ed., Academic Press, 2003.

[102] J. Delatorre, G. Baud, J.-J. Bzian, S. Blanco, C. Caliot, J.-F. Cornet, C. Coustet, J. Dauchet, M. El Hafi, V. Eymet, R. Fournier, J. Gautrais, O. Gourmel, D. Joseph, N. Meilhac, A. Pajot, M. Paulin, P. Perez, B. Piaud, M. Roger, J. Rolland, F. Veynandt, S. Weitz, Monte carlo advances and concentrated solar applications, Solar Energy 103 (0) (2014) 653-681.

[103] M. Roger, S. Blanco, M. El Hafi, R. Fournier, Monte carlo estimates of domain-deformation sensitivities, Physical Review Letters 95 (18) (2005) 180601.

[104] E. W. Weisstein, CRC concise encyclopedia of mathematics, 2nd ed., Chapman and Hall / CRC, 2003.

[105] A. Mugnai, W. J. Wiscombe, Scattering from nonspherical chebyshev particles. i: cross sections, single-scattering albedo, asymmetry factor and backscattered fraction, Applied Optics 25 (1986) 1235-1244.

[106] M. I. Mishchenko, L. D. Travis, Capabilities and limitations of a current fortran implementation of the T-matrix method 
for randomly oriented, rotationally symmetric scatterers, Journal of Quantitative Spectroscopy and Radiative Transfer 60 (1998) 309-324.

[107] J. Gielis, A generic geometric transformation that unifies a wide range of natural and abstract shapes, American Journal of Botany 90 (3) (2003) 333-338.

\section{Appendix A. Schiff's approximation}

The purpose of the present appendix is to transpose the Schiff approximation from quantum mechanics to electromagnetism, and then to derive our simplified behaviour of the differential scattering cross-section at large angles (further details on the Monte Carlo implementation of the approximation are developed in $[36])$.

The Schiff approximation was developed for the study of scattering of high-energy electrons from heavy nuclei. In his seminal article [81], L.I. Schiff addresses the wave function $\psi$ solution of the Schrödinger equation

$$
\left(\nabla^{2}+k_{e}^{2}-U\right) \psi=0
$$

with asymptotic functional form

$$
\psi \rightarrow \exp \left(i \mathbf{k}_{i} \cdot \mathbf{r}\right)+\frac{\exp \left(i k_{e} r\right)}{r} f\left(\mathbf{e}_{s}, \mathbf{e}_{i}\right)
$$

where $\frac{\hbar^{2} k^{2}}{2 m}$ is the kinetic energy of the incident particle and $\frac{\hbar^{2} U}{2 m}=V$ is the energy of the scattering potential. This study is conducted under the following assumptions:

(1) $|V| \ll\left(\mathcal{E}-m c^{2}\right)=\frac{\hbar^{2} k^{2}}{2 m}$

(2) $k_{e} a \gg 1$, where $a$ is the characteristic length corresponding to a significant variation of the potential $U$.

The transposition to our study is direct (within the scalar description of electromagnetic wave propagation [28]): we address the electric field $E$ solution of the Helmholtz equation

$$
\left(\nabla^{2}+k_{e, \nu}^{2} m_{r, \nu}^{2}\right) E=0
$$

with asymptotic functional form (far field zone)

$$
E \rightarrow \exp \left(i \mathbf{k}_{i} \cdot \mathbf{r}\right)+\frac{\exp \left(-i k_{e, \nu} r\right)}{-i r} S\left(\mathbf{e}_{s}, \mathbf{e}_{i}\right)
$$

where $S$ is the scattering amplitude. The solutions obtained in [81] (as well as their demonstration) can therefore be used in our context by considering $U=-k_{e, \nu}^{2}\left(m_{r, \nu}^{2}-1\right), S\left(\mathbf{e}_{s}, \mathbf{e}_{i}\right)=\frac{1}{-i} f\left(\mathbf{e}_{s}, \mathbf{e}_{i}\right)$, and replacing $i$ by $-i$ in all the expressions (in order to respect our convention for the complex amplitudes, i.e for the Fourier transform). In this transposition, the Schiff approximation corresponds to the following assumptions:

(a) assumption (1) corresponds to low refractive-index contrasts

$$
\left|m_{r, \nu}^{2}-1\right| \ll 1
$$

(b) assumption (2) corresponds to large particles compared to the wavelength (i.e large size parameters $\left.x=k_{e, \nu} a\right)$

$$
x \gg 1
$$

where $a$ is the characteristic dimension of the particle (its radius for a sphere).

Moreover, considering low refractive index contrasts, we retain (see [29]):

$$
m_{r, \nu}^{2}-1 \simeq 2\left(m_{r, \nu}-1\right)
$$

We therefore obtain the validity conditions and the expressions of the scattering amplitude given in Eqs. 13 and 16 of the present article. 
We hereafter focus on the expression of the differential scattering cross-section

$$
W_{s, \nu}\left(\mathbf{e}_{s} \mid \mathbf{e}_{i}\right)=\int_{4 \pi} d \mathbf{e}_{o} p_{\mathbf{E}_{o}}\left(\mathbf{e}_{o}\right) \int_{0}^{\infty} d r_{e q} p_{R_{e q}}\left(r_{e q}\right)\left|\hat{S}_{\nu}\left(\mathbf{e}_{s}, \mathbf{e}_{i} ; \mathbf{e}_{o}, r_{e q}\right)\right|^{2}
$$

for the large scattering angles, that is to say for $\hat{S}_{\nu}$ in Eq. 16:

$$
\hat{S}_{\nu}=\frac{i k_{e, \nu}^{2}}{2 \pi}\left(m_{r, \nu}-1\right) \int_{\mathcal{D}} d \mathbf{r} t(\mathbf{r}) h(\mathbf{r})
$$

where we introduce here the functions

$$
t(\mathbf{r})=\exp \left\{-k_{e, \nu} \kappa_{r, \nu} l^{t o t}(\mathbf{r})\right\}
$$

and

$$
h(\mathbf{r})=\exp \left\{i\left[\mathbf{q} \cdot \mathbf{r}-k_{e, \nu}\left(n_{r, \nu}-1\right) l^{t o t}(\mathbf{r})\right]\right\}
$$

with $l^{\text {tot }}(\mathbf{r})=l^{\text {in }}\left(\mathbf{r}, \mathbf{e}_{i}\right)+l^{\text {out }}\left(\mathbf{r}, \mathbf{e}_{s}\right)$ (see Fig. 3.b). At this stage, one has to notice that some effects due to the vectorial nature of the electromagnetic fields, that can be ignored at small scattering angles, has to be accounted for at large angles. In the present context (i.e radiative properties calculation for incoherent nonpolarized incident fields) these effects come down to the multiplication of $\left|\hat{S}_{\nu}\right|^{2}$ by the pre-factor $\frac{\cos ^{2}\left(\theta_{s}\right)+1}{2}$ (as for the Rayleigh-Gans approximation [29]), which leads to:

$$
\left|\hat{S}_{\nu}\right|^{2}=\frac{k_{e, \nu}^{4}}{4 \pi^{2}}\left|m_{r, \nu}-1\right|^{2} \frac{\cos ^{2}\left(\theta_{s}\right)+1}{2}\left|\int_{\mathcal{D}} d \mathbf{r} t(\mathbf{r}) h(\mathbf{r})\right|^{2}
$$

In order to derive our simplified behaviour of $W_{s, \nu}$ at large angles, we first write the square modulus of $\hat{S}_{\nu}$ as an integral over two locations $\mathbf{r}_{1}$ and $\mathbf{r}_{2}$ of the projected surface $\mathcal{P}$ :

$$
\begin{aligned}
\left|\hat{S}_{\nu}\right|^{2}= & \operatorname{Re}^{2}\{S\}+\operatorname{Im}^{2}\{S\} \\
= & \frac{k_{e, \nu}^{4}}{4 \pi^{2}}\left|m_{r, \nu}-1\right|^{2} \frac{\cos ^{2}\left(\theta_{s}\right)+1}{2} \int_{\mathcal{D}} d \mathbf{r}_{1} \int_{\mathcal{D}} d \mathbf{r}_{2} t\left(\mathbf{r}_{1}\right) t\left(\mathbf{r}_{2}\right) \\
& \times\left[\operatorname{Re}\left\{h\left(\mathbf{r}_{1}\right)\right\} \operatorname{Re}\left\{h\left(\mathbf{r}_{2}\right)\right\}+\operatorname{Im}\left\{h\left(\mathbf{r}_{1}\right)\right\} \operatorname{Im}\left\{h\left(\mathbf{r}_{2}\right)\right\}\right] \\
= & \frac{k_{e, \nu}^{4}}{4 \pi^{2}}\left|m_{r, \nu}-1\right|^{2} \frac{\cos ^{2}\left(\theta_{s}\right)+1}{2} \int_{\mathcal{D}} d \mathbf{r}_{1} \int_{\mathcal{D}} d \mathbf{r}_{2} t\left(\mathbf{r}_{1}\right) t\left(\mathbf{r}_{2}\right) \cos \left[h\left(\mathbf{r}_{1}\right)-h\left(\mathbf{r}_{2}\right)\right]
\end{aligned}
$$

with

$$
t\left(\mathbf{r}_{1}\right) t\left(\mathbf{r}_{2}\right)=\exp \left\{-k_{e, \nu} \kappa_{r, \nu} \Sigma l^{t o t}\right\}
$$

and

$$
\cos \left[h\left(\mathbf{r}_{1}\right)-h\left(\mathbf{r}_{2}\right)\right]=\cos \left[k_{e, \nu}\left(2 \sin \frac{\theta_{s}}{2} \hat{\mathbf{q}} \cdot \rho-\left(n_{r, \nu}-1\right) \Delta l^{t o t}\right)\right]
$$

where $\hat{\mathbf{q}}=\frac{\mathbf{q}}{\|\mathbf{q}\|}$ is the unit vector associated with $\mathbf{q}, \rho=\mathbf{r}_{1}-\mathbf{r}_{2}, \Sigma l^{\text {tot }}=l^{\text {tot }}\left(\mathbf{r}_{1}\right)+l^{\text {tot }}\left(\mathbf{r}_{2}\right)$ and $\Delta l^{\text {tot }}=$ $l^{\text {tot }}\left(\mathbf{r}_{1}\right)-l^{\text {tot }}\left(\mathbf{r}_{2}\right)$. Then, qualitatively applying the principle of the stationary phase approximation (that lies at the root of the Schiff approximation) we notice that the main contribution to Eq. A.5 comes from the integration domain such that $\|\rho\|<\frac{1}{k_{e, \nu} 2 \sin \left(\theta_{s} / 2\right)}$. Indeed, outside of this region the integrand rapidly oscillates around zero as $\rho$ variates because:

- $2 \sin \frac{\theta_{s}}{2} \hat{\mathbf{q}} \cdot \rho$ dominates the argument of the cosine in Eq. A.10,

- when $\mathbf{r}_{1}$ and $\mathbf{r}_{2}$ are distant enough $\left(\|\rho\|\right.$ is large) then $k_{e, \nu} \Delta l^{t o t} \in\left[0, k_{e, \nu} a\right]$ while at the opposite, when $\mathbf{r}_{1}=\mathbf{r}_{2}, k_{e, \nu} \Delta l^{t o t}=0$ 
If we neglect the effect of the boundary of $\mathcal{D}$, this stationary phase region corresponding to $\mathbf{r}_{1}=\mathbf{r}_{2}$ has a volume $\frac{4 \pi}{3\left(k_{e, \nu} 2 \sin \theta_{s} / 2\right)^{3}}$ regardless of the location $\mathbf{r}_{1}$, and since $\rho=\mathbf{0}, \Sigma l^{\text {tot }}=2 l^{\text {tot }}\left(\mathbf{r}_{1}\right)$ and $\Delta l^{\text {tot }}=0$. Thus, we obtain the simplification:

$$
\begin{aligned}
W_{s}\left(\theta_{s}\right) \propto \frac{1}{\left(\sin \theta_{s} / 2\right)^{3}} \frac{1+\cos ^{2} \theta_{s}}{2} & \int_{4 \pi} d \mathbf{e}_{o} p_{\mathbf{E}_{o}}\left(\mathbf{e}_{o}\right) \int_{0}^{\infty} d r_{e q} p_{R_{e q}}\left(r_{e q}\right) \\
& \times \int_{\mathcal{D}\left(\mathbf{e}_{o}, r_{e q}\right)} d \mathbf{r}_{1} \exp \left\{-k_{e, \nu} \kappa_{r, \nu} 2 l^{t o t}\left(\mathbf{r}_{1} ; \mathbf{e}_{o}, r_{e q}\right)\right\}
\end{aligned}
$$

Moreover, for the studied situations, we recorded variations of the term

$$
\int_{4 \pi} d \mathbf{e}_{o} p_{\mathbf{E}_{o}}\left(\mathbf{e}_{o}\right) \int_{0}^{\infty} d r_{e q} p_{R_{e q}}\left(r_{e q}\right) \int_{\mathcal{D}\left(\mathbf{e}_{o}, r_{e q}\right)} d \mathbf{r}_{1} \exp \left\{-k_{e, \nu} \kappa_{r, \nu} 2 l^{t o t}\left(\mathbf{r}_{1} ; \mathbf{e}_{o}, r_{e q}\right)\right\}
$$

as function of $\theta_{s}$ that are negligible compared to the variations of the first term in Eq. A.11. Therefore, we retain the following simplified behaviour for the variation of $W_{s}$ as function of $\theta_{s}$ at large angles, regardless of the scatterer geometry:

$$
W_{s}\left(\theta_{s}\right) \propto \frac{1}{\left(\sin \theta_{s} / 2\right)^{3}} \frac{1+\cos ^{2} \theta_{s}}{2}
$$

We notice that this mathematical expression is also encountered in the solution of $W_{s}$ for spheroids and cylinders with the Rayleigh-Gans approximation. 